\title{
EFFECTIVE BASE POINT FREENESS
}

\author{
DAISUKE Matsushita
}

\begin{abstract}
It is an interesting problem to know when the adjoint bundle $K_{X}+m L$ is free or very ample. Recently Ein-Lazarsfeld states very explicit numerical conditions about $L$ such that $K_{X}+L$ becomes free on a smooth 3 -fold. In this paper, the author wants to enlarge their results on a singular 3-fold.
\end{abstract}

\section{Introduction} 3-fold.

The purpose of this paper is to enlarge Ein-Lazarsfeld's result on a singular

It is an interesting problem when adjoint bundle $K_{X}+m L$ or pluricanonical system is free or very ample. Reider shows in [11] the following theorem:

THEOREM 1. Let $S$ be a smooth surface and $L$ be a nef Cartier divisor on S. Assume that:

(1) $L^{2} \geqq 5$,

(2) $L \cdot C \geqq 2$ for all curves on $S$.

Then the adjoint bundle $K_{S}+L$ is free.

From the above theorem, we can deduce the adjoint bundle $K_{S}+m L$ is free if $m \geqq 3$. Also Sakai shows in [12] that the similar statement holds on a normal surface. In higher dimension, Fujita conjectured in [4] the adjoint bundle $K_{X}+m L$ is free if $m \geqq \operatorname{dim} X+1$ and very ample if $m \geqq \operatorname{dim} X+2$. EinLazarsfeld got in [2] the following theorem:

THEOREM 2. Let $X$ be a smooth 3-fold and $L$ be a nef Cartier divisor. Fix one point $x \in X$. If $L$ satisfies the following criterion, the adjoint bundle $K_{X}+L$ is free at $x$.

(1) $L^{3} \geqq 92$,

(2) $L^{2} \cdot S \geqq 7$, for the all surfaces $S$ such that $x \in S$,

(3) $L \cdot C \geqq 3$, for the all curves $C$ such that $x \in C$.

By the above theorem, $K_{X}+m L$ is free if $m \geqq 5$. We want to enlarge the

Received October 11, 1994 ; revised January 9, 1995. 
above result on a singular 3 -fold, because it is natural to consider a variety which has some mild singularities by minimal model program. Recently EinLazarsfeld-Masek states the freeness of adjoint bundle on the terminal 3-fold in [3]. Our results are different from the above results at two points. First, we work on the variety which has only log-terminal singularities. Second, we consider the nef Cartier divisor $L$ such that $L-\left(K_{X}+\Delta\right)$ satisfies some numerical conditions. It will not be suitable to consider the adjoint bundle on a singular 3 -fold, since the canonical divisor is not a Cartier divisor in general. In such situation, Kollár shows in [10] the following theorem:

THEOREM 3. Let $X$ be a n-dimensional normal projective variety and $\Delta$ be an effective $\mathbf{Q}$-divisor on $X$. Assume that $(X, \Delta)$ has only log-terminal singularities. Let $L$ be a nef Cartier divisor such that $a L-\left(K_{X}+\Delta\right)$ is nef and big for some integer $a$. Then $(2(a+n)(n+2) !) L$ is free.

An analog of the conjecture of Fujita says that $(a+n+1) L$ is free. Kollár's result gave explicit estimates in all dimension, but it is far from above estimation. So we guess if we use the technique of Ein-Lazarsfeld's, we can do some improvement of Kollár's result in dimension 3, and we obtain the following theorems.

THEOREM 4. Let $X$ be a normal 3-fold and $\Delta$ be an effective $\boldsymbol{Q}$-divisor. Assume that $(X, \Delta)$ has only log-terminal singularities. Let $L$ be a nef Cartier divisor on $X$ such that $a L-\left(K_{X}+\Delta\right)$ is nef and big for some rational number $a>0$. Then $n L$ is free if $n>a+11 / 2,(n \in \boldsymbol{N})$.

THEOREM 5. Let $X$ and $\Delta$ are same objects in above Theorem. Assume that $(X, \Delta)$ has only weak log-terminal singularities and $X$ is $\boldsymbol{Q}$-factorial. Let $L$ be $a$ nef Cartier divisor on $X$ such that $a L-\left(K_{X}+\Delta\right)$ is ample for some rational number $a>0$. Then $n L$ is free if $n>a+11 / 2,(n \in \boldsymbol{N})$.

Similary Ein-Lazarsfeld-Masek [3], we can obtain the following result about a pluricanonical system.

THEOREM 6. Let $X$ be a minimal 3-fold of general type and $r$ be an index of $X$. Then

(1) $6 K_{X}$ is free if $r=1$,

(2) $m r K_{X}$ is free of

$$
m>2+\frac{1}{r}+\frac{7 \sqrt[3]{r}}{2 r} \quad r \geqq 2 .
$$

By the above theorem, we can deduce that $5 r K_{X}$ is free for $r \geqq 2$. If $r \geqq 4$ then $4 r K_{X}$ is free and if $r \geqq 9$ then $3 r K_{X}$ is free. Ein-Lazarsfeld-Masek proved in [3] that $10 K_{X}$ is free if $r=1,7 r K_{X}$ is free if $r \geqq 2,5 r K_{X}$ is free if $r \geqq 3$ and $2 r K_{X}$ is free if $r \geqq 27$. 


\section{The outline of the proof of theorems}

Fix one point $x$ on $X$. We construct a smooth variety $Y$ and a birational morphism $f: Y \rightarrow X$ depending on the type of singularity at $x$. According to the argument of Kawamata-Reid-Shokurov, we show the restriction map

$$
H^{0}\left(Y, f^{*} L-N\right) \longrightarrow H^{0}\left(E,\left.\left(f^{*} L-N\right)\right|_{E}\right)
$$

is surjective, where $N$ is an effective divisor such that $f^{-1}(x) \cap N=\emptyset$ and $E$ is a surface on $Y$ such that $x \in f(E)$. Then we will show there is a section $s \in$ $H^{0}\left(E,\left.\left(f^{*} L-N\right)\right|_{E}\right)$ which is non-vanishing at some point of $f^{-1}(x)$.

We distinguish three cases, according to $\operatorname{dim} f(E)=0,1,2$. In the case $\operatorname{dim} f(E)=0, \mathcal{O}_{E}\left(f^{*} L-N\right) \cong \mathcal{O}_{E}$ because $x \notin f(N)$. Thus we are done. In the case $\operatorname{dim} f(E)=1$, we prove the restriction map $H^{0}\left(E,\left(f^{*} L-N\right)_{E}\right) \rightarrow H^{0}\left(Z,\left.\left(f^{*} L-N\right)\right|_{Z}\right)$ is surjective, where $Z$ is a fibre of $\left.f\right|_{E}$ such that $Z \cap N=\emptyset$. Then $\mathcal{O}_{Z}(f * L-N)$ $\cong \mathcal{O}_{Z}$ and we are done. In the case $\operatorname{dim} f(E)=2$, we will produce the required section by the following theorem:

THEOREM 7. Let $S$ be a normal projective surface and $\Delta$ be an effective $\boldsymbol{Q}$ divisor. Assume that $(S, \Delta)$ has only log-terminal singularities. Fix one point $x$ on $S$. Let $Q$ be a Cartier divisor on $S$ which satısfies the following conditions:

(1) $M:=Q\left(K_{S}+\Delta\right)$ is nef and big,

(2) $M^{2}>4$,

(3) $M \cdot C>2$ for the all curves $x \in C$.

Then $x_{0} \notin \mathrm{Bs}|Q|$.

This Theorem is an extension of Theorem 2.1 in [2]. In Theorem 2.1 in [2], it is assumed that $S$ has only rational double points.

In this paper, $\S 2$ devoted to prove two elementary lemmas which used later. In $\S 3$, we prove Theorem 7 . The statement of main theorem appears in $\S 4$, where we also introduce Kawamata-Reid-Shokurov argument. We will show the existence of desirable section according to $\operatorname{dim} f(E)$ in $\S 5$ and will construct a smooth variety $Y$ and a birational morphism $f$ depending on the type of singularity at $x$ in $\S 6$.

Achnowledgement. The author would like to express his hearty thanks to Professor N. Nakayama for his useful advice. The author would like to thanks Professor J. Kollár, R. Lazarsfeld, and N.I. Shepard-Barron for their useful advice. By their advice, the author can improve Theorem 6 .

\section{Preliminary}

We work throughout over the complex numbers $\boldsymbol{C}$. Notation and terminology is same as in [7]. In this section, we prove two elementary lemmas which need the proof of Theorems. First we define a multiplicity of $\boldsymbol{Q}$-Cartier 
divisor $D$ at a point $x$.

DEFINITION 1. For an effective Cartier divisor $D$ on a projective variety $X$ and a point $x \in X$, define a natural number $\nu_{x}(D)$ by

$$
\nu_{x}(D):=\max \left\{n \in \boldsymbol{N} \mid \mathcal{O}_{X}(-D) \subset m_{x}^{n}\right\},
$$

where $m_{x}$ is a maximal ideal of $\mathcal{O}_{X}, x$. For an effective $\boldsymbol{Q}$-Cartier $\boldsymbol{Q}$-Weil divisor $D^{\prime}$ on $X$, define a rational number $\nu_{x}\left(D^{\prime}\right)$ by

$$
\nu_{x}\left(D^{\prime}\right):=\frac{1}{p} \nu_{x}\left(p D^{\prime}\right)
$$

where $p$ is a natural number such that $p D^{\prime}$ is a Cartier divisor.

Then we show the following lemma.

LEMMA 1. Let $X$ be a projective variety of dimension $d$ and $M$ be a nef and big $\boldsymbol{Q}$-Cartier divisor on $X$ such that $M^{d}>\boldsymbol{\alpha}^{d}$. Fix a point $x$ on $X$ such that mult $_{x} X=a$. Then there is an effective $Q$-divisor $B$ on $X$ such that $B \sim_{Q} M$ and

$$
\nu_{x}(B)>\frac{\alpha}{\sqrt[d]{a}(1-\sigma)} 0<\sigma \ll 1 .
$$

Proof. Consider the following exact sequence:

$$
0 \longrightarrow H^{0}\left(X, \mathcal{O}_{X}\left(m M \otimes m_{x}^{l}\right)\right) \longrightarrow H^{0}\left(X, \mathcal{O}_{X}(m M)\right) \longrightarrow H^{0}\left(X, \mathcal{O}_{X} / m_{x}^{l}\right) .
$$

By Kodaira's Lemma, we can choose an ample divisor $A$, an effective divisor $D$, and small numbers $0<\delta, \sigma \ll 1$ such that $M \sim_{Q} A+\delta D, A^{d}>(\alpha /(1-\sigma))^{d}$. For a sufficiently large number $m$ such that $m M$ and $m(M-\delta D)$ are Cartier,

$$
h^{0}(m M) \geqq h^{0}(m(M-\delta D))=\frac{A^{d}}{d !} m^{d}+(\text { lower terms of } m)>\frac{A^{d}-\varepsilon}{d !} m^{d},
$$

where $0<\varepsilon \ll \sigma \ll 1$. On the other hand,

$$
h^{0}\left(\mathcal{O}_{X} / m_{x}^{l}\right)=a \frac{a}{d !} l^{d}+(\text { lower terms of } l)<\frac{a+\varepsilon}{d !} l^{d}
$$

for a sufficiently large $l$. If we choose a suitable large $m$, there exists an integer $l$ which satisfy the following inequality,

$$
m \sqrt[d]{A^{d}-\varepsilon}>(\sqrt[d]{a+\varepsilon})^{l}>\frac{m \alpha}{1-\sigma} .
$$

For these $m$ and $l, h^{0}(m M)>h^{0}\left(\mathcal{O}_{X} / m_{x}^{l}\right)$. So we can choose a section $t \in H^{0}(m M)$ such that $\nu_{x}(\operatorname{div}(t)) \geqq l$. If we put $B:=(1 / m) \operatorname{div}(t)$ then the assertion of lemma follows.

LEMMA 2. Let $S$ be a normal surface and $M$ be a nef and big $\mathbf{Q}$-Cartier 
divisor on $S$. Fix one point $x_{0}$ on $S$. Assume that $\left(S, x_{0}\right)$ is a normal quotient surface singularity and $M^{2}>\left(\sigma_{2}\right)^{2}$. Let $\pi: S_{1} \rightarrow S$ be a minimal resolution of $\left(S, x_{0}\right)$ and $Z$ be a rational curve such that $Z \subset \pi^{-1}\left(x_{0}\right)$. Then there is an effective $\boldsymbol{Q}$-divisor $B$ on $S_{1}$ which satisfies the following two conditions:

(1) $B \sim_{Q} \pi * M$,

(2) $\quad B-\tau Z \geqq 0, \quad \tau>\frac{\sigma_{2}}{\sqrt{-Z^{2}}}, \quad \tau \in \boldsymbol{Q}$.

Proof. Consider the following exact sequence:

$$
\begin{aligned}
0 \longrightarrow & H^{0}\left(S_{1}, \mathcal{O}_{S_{1}}\left(m \pi^{*} M-(k+1) Z\right)\right) \\
& \longrightarrow H^{0}\left(S_{1}, \mathcal{O}_{S_{1}}\left(m \pi^{*} M-k Z\right)\right) \longrightarrow H^{0}\left(Z, \mathcal{O}_{Z}\left(m \pi^{*} M-k Z\right)\right),
\end{aligned}
$$

where $m$ is an integer such that $m \pi * M$ become a Cartier divisor. We obtain the following inequality,

$$
h^{0}\left(S_{1}, \mathcal{O}_{S_{1}}(m \pi * M-(k+1) Z)\right) \geqq h^{0}\left(S_{1}, \mathcal{O}_{S_{1}}(m \pi * M-k Z)\right)-h^{0}\left(S_{1}, \mathcal{O}_{Z}(-k Z)\right) .
$$

By Riemann-Roch formula,

$$
h^{0}\left(Z, \mathcal{O}_{Z}(-k Z)\right)-h^{0}\left(Z, \omega_{Z} \otimes \mathcal{O}_{Z}(k Z)\right)=\operatorname{deg} \mathcal{O}_{Z}(-k Z)+1-g(Z) .
$$

Since $Z^{2}<0$ and $g(Z)=0, h^{0}\left(Z, \mathcal{O}_{Z}(-k Z)\right)=-k Z^{2}+1$. Thus

$$
h^{0}\left(S_{1}, \mathcal{O}_{S_{1}}\left(m \pi^{*} M-(k+1) Z\right)\right) \geqq h^{0}\left(S_{1}, \mathcal{O}_{S_{1}}\left(m \pi^{*} M-k Z\right)\right)-\left(k Z^{2}+1\right) .
$$

From this inequality, we obtain

$$
h^{0}\left(S_{1}, \mathcal{O}_{S_{1}}\left(m \pi^{*} M-l Z\right)\right) \geqq h^{0}\left(S_{1}, \mathcal{O}_{S_{1}}\left(m \pi^{*} M\right)-\sum_{k=0}^{l-1}\left(-k Z^{2}+1\right) .\right.
$$

By Kodaira's Lemma, there are an ample divisor $A$ and an effective divisor $D$ such that $\pi^{*} M \sim_{Q} A+\varepsilon D, A^{2}>\left(\sigma_{2} /(1-\sigma)\right)^{2}$, where $\sigma$ and $\varepsilon$ are small numbers such that $0<\sigma, \varepsilon \ll 1$. If we take $m$ and $l$ large enough, we may assume $m \pi * M$, $m(\pi * M-\varepsilon D)$ are Cartier divisor and

$$
\begin{aligned}
h^{0}\left(S_{1}, \mathcal{O}_{S_{1}}\left(m \pi^{*} M\right)\right) & \geqq h^{0}\left(S_{1}, \mathcal{O}_{S_{1}}\left(m\left(\pi^{*} M-\varepsilon D\right)\right)\right) \\
& =\frac{m^{2}}{2} A^{2}+(\text { lower term of } m) \\
& >\frac{A^{2}-\varepsilon^{\prime}}{2} m^{2}, \\
\sum_{k=0}^{l-1}\left(-k Z^{2}+1\right) & =\frac{-Z^{2}}{2} l^{2}+(\text { lower term of } l) \\
& <\frac{-Z^{2}}{2}\left(l+\varepsilon^{\prime}\right)^{2}, \quad 0<\varepsilon^{\prime} \ll \sigma \ll 1 .
\end{aligned}
$$


Furthermore we may assume $m$ and $l$ satisfy the following inequality:

$$
m \sqrt{A^{2}-\varepsilon^{\prime}}>\left(l+\varepsilon^{\prime}\right) \sqrt{-Z^{2}}>\frac{m \sigma_{2}}{1-\sigma} .
$$

For these $m$ and $l$, there is a section $t \in H^{0}\left(m \pi^{*} M-l Z\right)$. Let $B:=(1 / m)(\operatorname{div}(t)$ $+l Z)$. Then the assertion of lemma follows.

\section{Effective base point freeness for surface}

THEOREM 7. Let $S$ be a normal projective surface and $\Delta$ be an effective $\boldsymbol{Q}$ divisor on $S$. Assume that $(S, \Delta)$ has only log-terminal singularities. Fix one point $x_{0}$ on $S$. Let $Q$ be a Cartier divisor on $S$ which satisfies the following condition:

(1) $M:=Q-\left(K_{S}+\Delta\right)$ is nef and big,

(2) $M^{2}>4$

(3) $M \cdot C>2$, for all curves $C \subset S$ such that $x_{0} \in C$.

Then $x_{0} \notin \mathrm{Bs}|Q|$.

Proof of Theorem. If $\left(S, x_{0}\right)$ is a smooth point or rational double point, Theorem 7 follows by the following theorem.

THEOREM 8 ([2] Theorem 2.1). Let $S$ be a normal projective surface which has only rational double points. Fix one point $x_{0}$ on $S$. Let $M$ be a nef and big $\boldsymbol{Q}$-divisor which has the following numerical criterion:

(1) $M^{2}>4$.

(2) $M \cdot C>2$, for all curves $C$ such that $x_{0} \in C$. Then $x_{0} \notin \mathrm{Bs} \mid K_{S}+\left\lceil M^{\top} \|\right.$.

Thus we may assume $\left(S, x_{0}\right)$ is quotient singularity. Let $\pi: S_{1} \rightarrow S$ be the minimal resolution. For an effective $\boldsymbol{Q}$-divisor $B$ such that $B \sim_{Q} \pi^{*} M$ and prime divisors $\left\{E_{i}\right\},(0 \leqq i \leqq m)$ on $S_{1}$ such that $\cup E_{\imath}=\operatorname{Supp} B \cup \pi^{-1}\left(x_{0}\right)$, we define rational numbers $\left\{b_{i}\right\}$ and $\left\{e_{i}\right\}$ by the following formulae:

Let

$$
\begin{gathered}
B=\sum b_{i} E_{\imath}, \\
K_{S_{1}} \sim_{Q} \pi^{*}\left(K_{S}+\Delta\right)+\sum e_{i} E_{\imath} .
\end{gathered}
$$

$$
c:=\min \left\{\frac{e_{i}+1}{b_{i}} \mid x_{0} \in \pi\left(E_{\imath}\right), b_{i}>0\right\}
$$

Claim 1. (1) $c b_{i}-e_{\imath} \geqq 0$, for any $i$.

(2) If $c b_{i}-e_{\imath}>1$, then $x_{0} \notin \pi\left(E_{\imath}\right)$.

Proof. Since $(S, \Delta)$ has only log-terminal singularities, we obtain $e_{\imath}>-1$ 
and $c>0$. Because $\pi$ is the minimal resolution, $e_{2} \leqq 0$. Thus $c b_{i}-e_{2} \geqq 0$.

(2) If $b_{i}>0$ and $x_{0} \in \pi\left(E_{\imath}\right)$, then $c \leqq\left(e_{i}+1\right) / b_{i}$. Thus $c b_{i}-e_{\imath} \leqq 1$. If $b_{i}=0$ and $c b_{i}-e_{\imath}>1$, then $e_{i}<-1$. But this contradicts the hypothesis that $(S, \Delta)$ has only log-terminal singularities.

We go back the proof of Theorem 7. Let $R:=\Sigma\left(c b_{i}-e_{\imath}\right) E_{\imath}$. By changing indices, we may assume the following:

(1) $c b_{i}-e_{\imath}=1$ and $E_{\imath}$ is not $\pi$-exceptional for $0 \leqq i \leqq m^{\prime}$.

(2) $c b_{i}-e_{i}=1$ and $E_{\imath}$ is $\pi$-exceptional for $m^{\prime}+1 \leqq i \leqq m_{1}$

(3) $c b_{i}-e_{2}>1$ for $m_{1}+1 \leqq i \leqq m_{2}$.

Let

$$
E^{\prime}:=\sum_{i=0}^{m^{\prime}} E_{\imath}, \quad E^{\prime \prime}:=\sum_{\imath=m^{\prime}+1}^{m_{1}} E_{\imath} \text { and } N:=\sum_{\imath=m_{1}+1}^{m_{2}}\left[c b_{i}-e_{i}\right] E_{\imath} .
$$

We define

$$
T:=\pi^{*} Q-N-E^{\prime}-E^{\prime \prime}-K_{S_{1}}-\langle R\rangle
$$

Then

$$
T \sim_{Q}(1-c) M
$$

Proposition 1. For a suitable $B, c<1$ holds.

$T$ is nef and big by Proposition 1 . First we consider the case $E^{\prime \prime} \neq 0$. We need the following lemma.

LEMMA 3 ([2] Lemma 1.1, 2.4). Let $S$ be a smmoth surface and $M$ be a nef and big $\boldsymbol{Q}$-divisor on $S$. Then

(1) $H^{1}\left(S, K_{S}+\lceil M\urcorner\right)=0$.

(2) Let $\left\{E_{i}\right\}$ be curves on $S$ such that $\operatorname{ord}_{E_{i}}(\langle M\rangle)=0$ and $\left.M \cdot E_{\imath}\right\rangle 0$. Then

$$
H^{1}\left(S, K_{S}+\lceil M\urcorner+E_{1}+\cdots+E_{k}\right)=0 .
$$

By Lemma 3, we obtain

$$
H^{1}\left(S_{1}, \mathcal{O}_{S_{1}}\left(K_{S_{1}}+\lceil T\rceil+E^{\prime}\right)\right) \cong H^{1}\left(S_{1}, \mathcal{O}_{S_{1}}\left(\pi * Q-N-E^{\prime \prime}\right)\right)=0 .
$$

Thus the restriction map

$$
H^{0}\left(S_{1}, \mathcal{O}_{S_{1}}(\pi * Q-N)\right) \longrightarrow H^{0}\left(E^{\prime \prime}, \mathcal{O}_{E^{\prime \prime}}(\pi * Q-N)\right)
$$

is surjective. Since $E^{\prime \prime}$ is $\pi$-exceptional and $x_{0} \notin \pi(N)$ by Claim $1, \mathcal{O}_{E^{\prime \prime}}(\pi * Q-N)$ $\cong \mathcal{O}_{E^{\prime \prime}}$. Therefore we obtain a section $t \in H^{0}\left(S_{1}, \mathcal{O}_{S_{1}}\left(\pi^{*} Q-N\right)\right)$ which doesn't vanish at some points of $\pi^{-1}\left(x_{0}\right)$. Because $x_{0} \notin \pi(N)$, the existence of $t$ shows $x_{0} \notin \mathrm{Bs}|Q|$.

Next we consider the case $E^{\prime \prime}=0$. By Lemma 3,

$$
H^{1}\left(S_{1}, \mathcal{O}_{S_{1}}\left(K_{S_{1}}+\lceil T\urcorner+E^{\prime}-E_{0}\right)\right) \cong H^{1}\left(S_{1}, \mathcal{O}_{S_{1}}\left(\pi * Q-N-E_{0}\right)\right)=0 .
$$

Thus the restriction map 


$$
H^{0}\left(S_{1}, \mathcal{O}_{S_{1}}(\pi * Q-N)\right) \longrightarrow H^{0}\left(E_{0}, \mathcal{O}_{E_{0}}(\pi * Q-N)\right)
$$

is surjective.

Proposition 2. For a suitable $B, \Gamma T\urcorner \cdot E_{0}>1$.

Since $\lceil T\urcorner \cdot E$ is integer, $\lceil T] \cdot E \geqq 2$ by Proposition 2. Then $\operatorname{deg}\left(\mathcal{O}_{E_{0}}(\pi * Q-N)\right)$ $\geqq 2 p_{a}\left(E_{0}\right)$, because

$$
\left.\mathcal{O}_{E_{0}}(\pi * Q-N) \cong \mathcal{O}_{E_{0}}\left(K_{E_{0}}+\Gamma T\right\urcorner+E_{1}+\cdots+E_{m^{\prime}}\right) .
$$

Since $E_{0}$ is Gorenstein, $\mathcal{O}_{E_{0}}(\pi * Q-N)$ is globally generated (cf. [5]) and there is a section $t \in H^{0}\left(E_{0}, \mathcal{O}_{E_{0}}(\pi * Q-N)\right)$ such that $t(y) \neq 0$ for some point $y \in \pi^{-1}\left(x_{0}\right)$. Thus we can deduce $x_{0} \notin \mathrm{Bs}|Q|$. For the rest of the proof of Theorem 7, it is enough to show Proposition 1, 2. Now we will give a proof of Proposition 1, 2.

Proof of Proposition 1. Let $M^{2}=\left(\sigma_{2}\right)^{2}$ and $M \cdot C \geqq \sigma_{1}$ for all curves $C \subset S$ such that $x_{0} \in C$. Note that $\sigma_{2}>2$ and $\sigma_{1}>2$ by the assumption of Theorem 7 .

LEMMA 4. For a suitable $B$,

(1) $c<\frac{(n+1) \sqrt{3}}{(2 n+1) \sigma_{2}}$, when the dual graph of $\pi^{-1}\left(x_{0}\right)$ is of type $A_{n}$.

(2) $c<\frac{\sqrt{3}}{2 \sigma_{2}}$, when the dual graph of $\pi^{-1}\left(x_{0}\right)$ is of type $D_{n}$.

(3) $c<\frac{1}{\sigma_{2}}$, when the dual graph of $\pi^{-1}\left(x_{0}\right)$ is of type $E_{n}$.

We can obtain the assertion of Proposition 1 by the above lemma, because $\sigma_{2}>2$.

Proof of Lemma 4. We show (1) and (2) in parallel. By changing indices, we may assume $\pi^{-1}\left(x_{0}\right)=\sum_{\imath=1}^{n} E_{\imath}$. We define rational numbers $a_{\imath}$ by the formula $K_{S_{1}} \sim_{Q} \pi * K_{S}+\sum a_{i} E_{\imath}$.

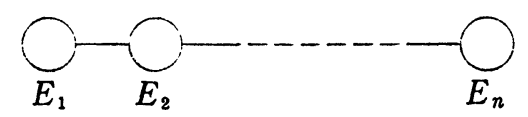

Figure 1. The dual graph of type $A_{n}$.

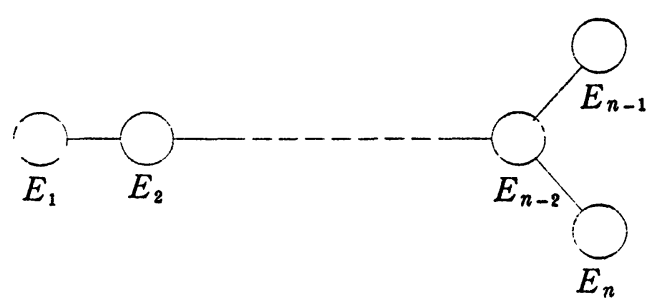

Figure 2. The dual graph of type $D_{n}$. 
ClaIm 2. (1) If the dual graph of $\pi^{-1}\left(x_{0}\right)$ is as in Figure 1, then

$$
a_{\imath} \leqq-1+\left(\frac{1}{\imath}+\frac{1}{n-i+1}\right)\left(\delta_{i}-\frac{i-1}{i}-\frac{n-i}{n-i+1}\right)^{-1}, \text { for any } i,
$$

where $\delta_{i}:=-\left(E_{\imath}\right)^{2}$.

(2) If the dual graph of $\pi^{-1}\left(x_{0}\right)$ is as in Figure 2, then

$$
a_{\imath} \leqq-1+\frac{1}{i}\left(\delta_{i}-\frac{i-1}{i}-1\right)^{-1}, \text { for any } \imath \text {, }
$$

where $\delta_{i}:=-\left(E_{\imath}\right)^{2}$.

First we shall show the assertion of Lemma 4 (1), (2) by assuming Claim 2. Let $E_{k}$ be the component which has the minimum self intersection number $\left(E_{k}\right)^{2}$ in $\left\{E_{i}\right\}$. By Lemma 2, we can take an effective $\boldsymbol{Q}$-divisor $B$ such that $B \sim_{Q} \pi * M$ and $B-\left(\sigma_{2} /\left(\sqrt{ } \delta_{k}(1-\sigma)\right)\right) E_{k} \geqq 0,(0<\sigma \ll 1)$. Then

$$
b_{k} \geqq \frac{\sigma_{2}}{\sqrt{\delta_{k}}(1-\sigma)} .
$$

If the dual graph of $\pi^{-1}\left(x_{0}\right)$ is of type $A_{n}$,

$$
e_{k} \leqq a_{k} \leqq-1+\left(\frac{1}{k}+\frac{1}{n-k+1}\right)\left(\delta_{k}-\frac{k-1}{k}-\frac{n-k}{n-k+1}\right)^{-1}, \text { for any } k
$$

Thus

$$
\begin{aligned}
c & \leqq \frac{e_{k}+1}{b_{k}} \\
& =\left(\frac{1}{k}+\frac{1}{n-k+1}\right)\left(\delta_{k}-\frac{k-1}{k}-\frac{n-k}{n-k+1}\right)^{-1} \frac{(1-\sigma) \sqrt{\delta_{k}}}{\sigma_{2}} \\
& \left.=\left(\frac{1}{k}+\frac{1}{n-k+1}\right)\left(\sqrt{\delta_{k}}-\frac{k-1}{k}-\frac{n-k}{n-k+1}\right) \frac{1}{\sqrt{\delta_{k}}}\right)^{-1} \frac{1-\sigma}{\sigma_{2}} .
\end{aligned}
$$

The function

$$
\delta_{k} \longmapsto\left(\sqrt{\delta_{k}}-\left(\frac{k-1}{k}+\frac{n-k}{n-k+1}\right) \frac{1}{\sqrt{\delta_{k}}}\right)^{-1} \frac{1-\sigma}{\sigma_{2}}
$$

is decreasing in $\delta_{k}$, and because $\delta_{k} \geqq 3$,

$$
\begin{aligned}
& \left(\frac{1}{k}+\frac{1}{n-k+1}\right)\left(\delta_{k}-\frac{k-1}{k}-\frac{n-k}{n-k+1}\right)^{-1} \frac{(1-\sigma) \sqrt{\delta_{k}}}{\sigma_{2}} \\
& \quad \leqq\left(\frac{1}{k}+\frac{1}{n-k+1}\right)\left(3-\frac{k-1}{k}-\frac{n-k}{n-k+1}\right)^{-1} \frac{(1-\sigma) \sqrt{3}}{\sigma_{2}} \\
& \quad<\frac{(n+1) \sqrt{3}}{(k(n-k+1)+n+1) \sigma_{2}} .
\end{aligned}
$$


Let $N(k):=k(n-k+1)$. Then $N(k) \geqq n$ because $1 \leqq k \leqq n$. Therefore

$$
c \leqq \frac{(n+1) \sqrt{3}}{(2 n+1) \sigma_{2}} .
$$

This completes the proof of Lemma 4 (1).

If the dual graph of $\pi^{-1}\left(x_{0}\right)$ is type $D_{n}$,

$$
e_{k} \leqq a_{k} \leqq-1+\frac{1}{k}\left(\delta_{k}-\frac{k-1}{k}-1\right)^{-1}
$$

Thus

$$
\begin{aligned}
c & \leqq \frac{e_{k}+1}{b_{k}} \\
& =\frac{1}{k}\left(\delta_{k}-\frac{k-1}{k}-1\right)^{-1} \frac{\sqrt{\delta_{k}}(1-\sigma)}{\sigma_{2}} \\
& =\frac{1}{k}\left(\sqrt{\delta_{k}}-\left(\frac{k-1}{k}+1\right) \frac{1}{\sqrt{\delta_{k}}}\right)^{-1} \frac{1-\sigma}{\sigma_{2}} .
\end{aligned}
$$

The function

$$
\delta_{k} \longmapsto \frac{1}{k}\left(\sqrt{\delta_{k}}-\left(\frac{k-1}{k}+1\right) \frac{1}{\sqrt{\delta_{k}}}\right)^{-1}
$$

is decreasing in $\delta_{k}$ and because $\delta_{k} \geqq 3$,

$$
\begin{aligned}
& \frac{1}{k}\left(\delta_{k}-\frac{k-1}{k}-1\right)^{-1} \frac{\sqrt{\delta_{k}(1-\sigma)}}{\sigma_{2}} \\
& \leqq \frac{1}{k}\left(3-\frac{k-1}{k}-1\right)^{-1} \frac{\sqrt{3(1-\sigma)}}{\sigma_{2}}=\frac{\sqrt{3(1-\sigma)}}{1+k} \\
& \leqq \frac{\sqrt{3(1-\sigma)}}{2 \sigma_{2}}<\frac{\sqrt{3}}{2 \sigma_{2}} .
\end{aligned}
$$

This completes the proof of Lemma 4 (2).

Proof of Claim 2. (1) We consider $K_{S_{1}} E_{\jmath}=\pi * K_{S} E_{j}+\Sigma a_{i} E_{i} E_{\jmath}$. Then we obtain the following linear equations :

$$
\begin{aligned}
& \delta_{1}-2=-\delta_{1} a_{1}+a_{2}, \\
& \delta_{k}-2=-\delta_{k} a_{k}+a_{k-1}+a_{k+1}, \quad(2 \leqq k \leqq n-1) \\
& \delta_{n}-2=-\delta_{n} a_{n}+a_{n-1} .
\end{aligned}
$$

Thus

$$
a_{1}=-1+P_{n}+\prod_{\jmath=1}^{n} P_{\jmath}
$$


and

$$
a_{k}=-1+\left(\prod_{j=1}^{k-1} Q_{j}+\prod_{j=1}^{n-k} P_{\jmath}\right)\left(\delta_{k}-P_{n-k}-Q_{k-1}\right)^{-1}, \quad k \neq 1,
$$

where

$$
\begin{aligned}
P_{\jmath} & :=\frac{1}{\delta_{n-\jmath+1}}-\frac{1}{\delta_{n-\jmath+2}}-\cdots-\frac{1}{\delta_{n}}, \quad(j \geqq 2), \\
P_{1} & :=\frac{1}{\delta_{n}} \\
Q_{\jmath} & :=\frac{1}{\delta_{j}}-\frac{1}{\delta_{j-1}}-\cdots-\frac{1}{\delta_{1}}, \quad(j \leqq 2), \\
Q_{1} & :=\frac{1}{\delta_{1}} .
\end{aligned}
$$

Because $\delta_{i} \geqq 2$,

$$
P_{\jmath} \leqq \frac{j}{j+1} \text { and } \quad Q_{\jmath} \leqq \frac{\jmath}{j+1}
$$

Thus we obtain

$$
\begin{aligned}
a_{1} & =-1+\frac{1}{\delta_{1}-P_{n-1}}+\frac{1}{\delta_{1}-P_{n-1}} \prod_{\jmath=1}^{n-1} P_{\jmath} \\
& \leqq-1+\frac{1}{\delta_{1}-(n-1) / n}+\frac{1}{\delta_{1}-(n-1) / n} \prod_{\jmath=1}^{n-1} \frac{\jmath}{j+1} \\
& =-1+\frac{n+1}{n \delta_{1}-n+1}
\end{aligned}
$$

and

$$
\begin{aligned}
a_{k} & \leqq-1+\left(\prod_{\jmath=1}^{k-1} \frac{\jmath}{\jmath+1}+\prod_{\jmath=1}^{n-k} \frac{\jmath}{\jmath+1}\right)\left(\delta_{k}-\frac{k-1}{k}-\frac{n-k}{n-k+1}\right) \\
& =-1+\left(\frac{1}{k}+\frac{1}{n-k+1}\right)\left(\delta_{k}-\frac{k-1}{k}-\frac{n-k}{n-k+1}\right), \quad(k \geqq 2) .
\end{aligned}
$$

(2) We consider $K_{S_{1}} E_{\jmath}=\pi * K_{S} E_{j}+\sum a_{i} E_{\imath} E_{\jmath}$. Then we obtain the following linear equations :

$$
\begin{aligned}
\delta_{1}-2 & =-\delta_{1} a_{1}+a_{2}, \\
\delta_{k}-2 & =-\delta_{k} a_{k}+a_{k-1}+a_{k+1}, \quad(2 \leqq k \leqq n-3), \\
\delta_{n-2}-2 & =-\delta_{n-2} a_{n-2}+a_{n-3}+a_{n-1}+a_{n}, \\
\delta_{j}-2 & =-\delta_{j} a_{j}+a_{n-2}, \quad(j=n-1, n) .
\end{aligned}
$$


Note that $\delta_{j}=2$ for $j=n-1$ or $j=n$. Thus

$$
\begin{aligned}
a_{1} & =-1+S_{n-2} \\
a_{k} & =-1+\left(\prod_{j=1}^{k-1} T_{j}\right)\left(\delta_{k}-T_{k-1}-S_{n-k-2}\right)^{-1}, \quad(2 \leqq k \leqq n-3) \\
a_{n-2} & =-1+\left(\prod_{j=1}^{n-3} T_{\jmath}\right)\left(\delta_{n-2}-T_{n-3}-1\right)^{-1},
\end{aligned}
$$

where

$$
\begin{aligned}
S_{\jmath} & :=\frac{1}{\delta_{n-\jmath-1}}-\frac{1}{\delta_{n-\jmath}}-\cdots-\frac{1}{\delta_{n-2}-1}, \quad(j \geqq 2), \\
S_{1} & :=\frac{1}{\delta_{n-2}-1}, \\
T_{j} & :=\frac{1}{\delta_{j}}-\frac{1}{\delta_{j-1}}-\cdots-\frac{1}{\delta_{1}}, \quad(j \geqq 2), \\
T_{1} & :=\frac{1}{\delta_{1}} .
\end{aligned}
$$

Because $\delta_{i} \geqq 2$,

$$
S_{\jmath} \leqq 1 \text { and } T_{\jmath} \leqq \frac{j}{j+1}
$$

Thus we obtain

$$
a_{1}=-1+\frac{1}{\delta_{1}-S_{n-3}} \leqq-1+\frac{1}{\delta_{1}-1}
$$

and

$$
\begin{aligned}
a_{k} & \leqq-1+\left(\prod_{j=1}^{k-1} \frac{j}{j+1}\right)\left(\delta_{k}-\frac{k-1}{k}-1\right)^{-1} \\
& =-1+\frac{1}{k}\left(\delta_{k}-\frac{k-1}{k}-1\right)^{-1}, \quad(k \geqq 2) .
\end{aligned}
$$

We have completed the proof of Claim 2 .

We go back the proof of Lemma 4. We consider (3). Define rational numbers $\left\{a_{i}\right\}$ by $K_{S_{1}} \sim_{Q} \pi * K_{S}+\sum a_{i} E_{\imath}$. We denote by $(\mu ; a, b, c ; d, e)$ the dual graph of type $E_{n}$ as in Figure 3. Then the classification of dual graphs of type $E_{n}$ listed in Table 1. By changing indices, we may assume $\left(E_{1}\right)^{2}=-\mu$. We can take an effective $\boldsymbol{Q}$-divisor $B$ such that $B \sim_{Q} \pi^{*} M$ and $B-\left(\sigma_{2} /(1-\sigma) \sqrt{\mu}\right) E_{1}, \quad(0<\sigma \ll 1)$ by Lemma 2. Considering $K_{S_{1}} E_{\jmath}=\pi * K_{S} E_{j}+$ $\sum a_{i} E_{i} E_{\jmath}, a_{1}$ is computed as in Table 2 . We can write $\sqrt{\mu}\left(a_{1}+1\right)$ as 


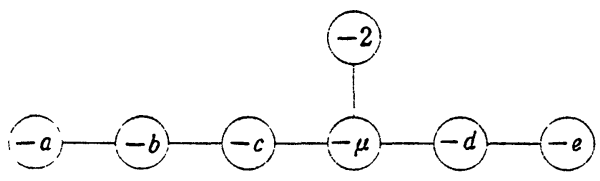

Figure 3. The dual graph of type $E_{n}$.

Table 1. List of dual graphs of type $E_{n}$.

\begin{tabular}{|c|c|}
\hline Type & \multicolumn{1}{|c|}{ dual graph } \\
\hline 1 & $\mu ; 2,2 ; 2,2$ \\
\hline 2 & $\mu ; 2,2 ; 3$ \\
\hline 3 & $\mu ; 3 ; 3$ \\
\hline 4 & $\mu ; 2,2 ; 2,2,2$ \\
\hline 5 & $\mu ; 3 ; 2,2,2$ \\
\hline 6 & $\mu ; 2,2 ; 4$ \\
\hline 7 & $\mu ; 3 ; 4$ \\
\hline 8 & $\mu ; 2,2 ; 2,2,2,2$ \\
\hline 9 & $\mu ; 3 ; 2,2,2,2$ \\
\hline 10 & $\mu ; 2,2 ; 3,2$ \\
\hline 11 & $\mu ; 3 ; 3,2$ \\
\hline 12 & $\mu ; 2,2 ; 2,3$ \\
\hline 13 & $\mu ; 3 ; 2,3$ \\
\hline 14 & $\mu ; 2,2 ; 5$ \\
\hline 15 & $\mu ; 3 ; 5$ \\
\hline
\end{tabular}

Table 2. List of $a_{1}$.

\begin{tabular}{|c|c|c|}
\hline Type & & \\
\hline 1 & $a_{1}=-1+1 /(6 \mu-11)$ & $\mu \geqq 3$ \\
\hline 2 & $a_{1}=-1+1 /(6 \mu-9)$ & $\mu \geqq 2$ \\
\hline 3 & $a_{1}=-1+1 /(6 \mu-7)$ & $\mu \geqq 2$ \\
\hline 4 & $a_{1}=-1+1 /(12 \mu-23)$ & $\mu \geqq 3$ \\
\hline 5 & $a_{1}=-1+1 /(12 \mu-19)$ & $\mu \geqq 2$ \\
\hline 6 & $a_{1}=-1+1 /(12 \mu-17)$ & $\mu \geqq 2$ \\
\hline 7 & $a_{1}=-1+1 /(12 \mu-13)$ & $\mu \geqq 2$ \\
\hline 8 & $a_{1}=-1+1 /(30 \mu-59)$ & $\mu \geqq 3$ \\
\hline 9 & $a_{1}=-1+1 /(30 \mu-49)$ & $\mu \geqq 2$ \\
\hline 10 & $a_{1}=-1+1 /(30 \mu-47)$ & $\mu \geqq 2$ \\
\hline 11 & $a_{1}=-1+1 /(30 \mu-37)$ & $\mu \geqq 2$ \\
\hline 12 & $a_{1}=-1+1 /(30 \mu-53)$ & $\mu \geqq 2$ \\
\hline 13 & $a_{1}=-1+1 /(30 \mu-43)$ & $\mu \geqq 2$ \\
\hline 14 & $a_{1}=-1+1 /(30 \mu-41)$ & $\mu \geqq 2$ \\
\hline 15 & $a_{1}=-1+1 /(30 \mu-31)$ & $\mu \geqq 2$ \\
\hline
\end{tabular}




$$
\sqrt{\mu}\left(a_{1}+1\right)=\frac{\sqrt{\mu}}{\alpha \mu-\beta}=\frac{1}{\alpha \sqrt{\mu}-(\beta / \sqrt{\mu})},
$$

where $\alpha$ and $\beta$ are positive integers. The function

$$
\mu \longmapsto \frac{1}{\alpha \sqrt{\mu}-(\beta / \sqrt{\mu})}
$$

is decreasing function in $\mu$. Then we can deduce $\sqrt{\mu}\left(a_{1}+1\right)<1$ by Table 2 . Thus

$$
c \leqq \frac{e_{1}+1}{b_{1}} \leqq \frac{a_{1}+1}{b_{1}} \leqq \frac{\left(a_{1}+1\right) \sqrt{\mu}(1-\sigma)}{\sigma_{2}}<\frac{1}{\sigma_{2}} .
$$

This completes the proof of Lemma 4 (3). We have now completed the proof of Lemma 4.

Proof of Proposition 2. We will prove Proposition 2 dividing into two cases.

CASE 1. The dual graph of $\pi^{-1}\left(x_{0}\right)$ is of type $A_{n},(n \geqq 3), D_{n}$ or $E_{n}$.

CASE 2. The dual graph of $\pi^{-1}\left(x_{0}\right)$ is of type $A_{n},(n \leqq 2)$.

In Case 1, Proposition 2 follows by the following Claim.

Claim 3. If the dual graph of $\pi^{-1}\left(x_{0}\right)$ is of type $A_{n},(n \geqq 3), D_{n}$ or $E$, then $c<1 / 2$.

Since $T \sim_{Q}(1-c) M$,

$$
T \cdot E_{0}=(1-c) M \cdot E_{0} \geqq(1-c) \sigma_{1} .
$$

By Claim 3

$$
T \cdot E_{0}>\left(1-\frac{1}{2}\right) \sigma_{1}=\frac{\sigma_{1}}{2}
$$

Thus we obtain

$$
\lceil T\urcorner \cdot E_{0} \geqq T \cdot E_{0}>\frac{\sigma_{1}}{2}>1
$$

because $\sigma_{1}>2$. This completes the proof of Proposition 2 in Case 1 .

Proof of Claim 3. If the dual graph of $\pi^{-1}\left(x_{0}\right)$ is of type $A_{n}, c<((n+1) \sqrt{3})$ $/(2 n+1) \sigma_{2}$ by Lemma 4 . The function

$$
n \longmapsto \frac{n+1}{2 n+1}
$$

is decreasing function in $n$. Hence

$$
c<\frac{(n+1) \sqrt{3}}{(2 n+1) \sigma_{2}}<\frac{4 \sqrt{3}}{7 \sigma_{2}}<\frac{4 \sqrt{3}}{14}<\frac{1}{2}
$$


by Lemma $4, n \geqq 3$ and $\sigma_{2}>2$.

If the dual graph of $\pi^{-1}\left(x_{0}\right)$ is of type $D_{n}$. By Lemma $4, c<\sqrt{3} /\left(2 \sigma_{2}\right)$. Since $\sigma_{2}>2$, we obtain $c<\sqrt{3} / 4<1 / 2$.

If the dual graph of $\pi^{-1}\left(x_{0}\right)$ is of type $E_{n}, c<1 / \sigma_{2}$ by Lemma 4 . Because $\sigma_{2}>2$, we can obtain $c<1 / 2$. Then we completed the proof of Claim 3 .

We go back the proof of Proposition 2. We consider Case 2. By changing indices, we may assume $\pi^{-1}\left(x_{0}\right)=\sum_{\imath=1}^{n} E_{\imath}$. Define rational numbers $\left\{a_{i}\right\}$ by the following formula $K_{S_{1}} \sim_{Q} \pi^{*} K_{S}+\sum a_{i} E_{\imath}$. Since $E^{\prime \prime}=0, c b_{i}-e_{i}<1$ for $1 \leqq i \leqq n$. So $\lceil T\urcorner-T \geqq \sum_{\imath=1}^{n}\left(c b_{i}-e_{\imath}\right) E_{\imath}$. Hence

$$
\lceil T\urcorner \cdot E_{0} \geqq T \cdot E_{0}+\left(\sum_{\imath=1}^{n}\left(c b_{i}-e_{\imath}\right) E_{\imath}\right) E_{0} .
$$

Because $x_{0} \in \pi\left(E_{0}\right), E_{\imath} \cdot E_{0}>0$ for some $i(1 \leqq i \leqq n)$. We obtain

$$
\left(\sum_{\imath=1}^{n}\left(c b_{i}-e_{\imath}\right) E_{\imath}\right) E_{0} \geqq\left(\sum_{\imath=1}^{n}-e_{i} E_{\imath}\right) E_{0} \geqq-\max \left\{e_{\imath} \mid 1 \leqq i \leqq n\right\} .
$$

Therefore

$$
\lceil T\urcorner \cdot E_{0} \geqq T \cdot E_{0}-\max \left\{e_{\imath} \mid 1 \leqq i \leqq n\right\} .
$$

ClAIM 4. (1) If $n=1$, then $\max \left\{e_{\imath} \mid 1 \leqq i \leqq n\right\} \leqq-1 / 3$.

(2) If $n=2$, then $\max \left\{e_{\imath} \mid 1 \leqq i \leqq n\right\} \leqq-1 / 5$.

Proof. (1) By considering $K_{S_{1}} E_{1}=\pi * K_{S} E_{1}+a_{1}\left(E_{1}\right)^{2}$, we obtain the linear equation :

$$
\delta_{1}-2=-a_{1} \delta_{1}
$$

where $\delta_{1}:=-\left(E_{1}\right)^{2}$. Thus $a_{1}=1+2 / \delta_{1}$. Because $\delta_{1} \geqq 3$,

$$
e_{1} \leqq a_{1} \leqq-1+\frac{2}{3}=-\frac{1}{3} \text {. }
$$

(2) By considering $K_{S_{1}} E_{\imath}=\pi^{*} K_{S} E_{i}+\sum_{j=1}^{2} a_{j} E_{j} E_{\imath}$, we obtain the linear equations :

$$
\begin{aligned}
& \delta_{1}-2=-a_{1} \delta_{1}+a_{2} \\
& \delta_{2}-2=-a_{2} \delta_{2}+a_{1},
\end{aligned}
$$

where $\delta_{i}:=-\left(E_{\imath}\right)^{2}$. Then we obtain

$$
\begin{aligned}
& a_{1}=-1+\frac{1}{\delta_{1}-\left(1 / \delta_{2}\right)}+\frac{1}{\delta_{1} \delta_{2}-1} \\
& a_{2}=-1+\frac{1}{\delta_{2}-\left(1 / \delta_{1}\right)}+\frac{1}{\delta_{1} \delta_{2}-1} .
\end{aligned}
$$

We may assume $\delta_{1} \geqq 3$ and $\delta_{2} \geqq 2$. Then 


$$
\frac{1}{\delta_{1}-\left(1 / \delta_{2}\right)} \leqq \frac{3}{5}, \quad \frac{1}{\delta_{2}-\left(1 / \delta_{1}\right)} \leqq \frac{2}{5} \text { and } \frac{1}{\delta_{1} \delta_{2}-1} \leqq \frac{1}{5} \text {. }
$$

Thus

$$
\max \left\{e_{\imath} \mid 1 \leqq i \leqq 2\right\} \leqq \max \left\{a_{\imath} \mid 1 \leqq i \leqq 2\right\} \leqq-\frac{1}{5} .
$$

This completes the proof of Claim 3 .

We go back to the proof of Proposition 2 in Case 2. By Claim 4

$$
\begin{aligned}
\Gamma T\urcorner \cdot E_{0} & \geqq T \cdot E_{0}-\max \left\{e_{i} \mid 1 \leqq i \leqq n\right\} \\
& =(1-c) M \cdot E_{0}+\frac{1}{3} \quad(n=1), \\
& =(1-c) M \cdot E_{0}+\frac{1}{5} \quad(n=2) .
\end{aligned}
$$

Then by Lemma 4 ,

$$
c<\frac{(n+1) \sqrt{3}}{(2 n+1) \sigma_{2}}
$$

We obtain

$$
\begin{aligned}
(1-c) M \cdot E_{0} & >\left(1-\frac{2 \sqrt{3}}{3 \sigma_{2}}\right) \sigma_{1} \quad(n=1), \\
& >\left(1-\frac{3 \sqrt{3}}{5 \sigma_{2}}\right) \sigma_{1} \quad(n=2) .
\end{aligned}
$$

Because $\sigma_{1}>2$ and $\sigma_{2}>2$,

$$
\begin{aligned}
(1-c) M \cdot E_{0} & >\left(2-\frac{2 \sqrt{3}}{3}\right) \quad(n=1), \\
& >\left(2-\frac{3 \sqrt{3}}{5}\right) \quad(n=2) .
\end{aligned}
$$

Therefore

$$
\begin{aligned}
\lceil T\urcorner \cdot E_{0} & >\left(2-\frac{2 \sqrt{3}}{3}\right)+\frac{1}{3}=\frac{7-2 \sqrt{3}}{3}>1 \quad(n=1), \\
> & \left(2-\frac{3 \sqrt{3}}{5}\right)+\frac{1}{5}=\frac{11-3 \sqrt{3}}{5}>1 \quad(n=2) .
\end{aligned}
$$

We have completed the proof of Proposition 2.

Now we have completed the proof of Theorem 7 .

Q.E.D. 


\section{Statement of main theorem}

TheOREM 9. Let $X$ be a normal projective 3-fold and $\Delta$ be an effective $\boldsymbol{Q}$ divisor on $X$. Assume that $(X, \Delta)$ has only log-terminal singularities. Let $X_{0}$ be a normal projective variety and $g: X \rightarrow X_{0}$ be a projective morphism. Let $Q$ be a Cartier divisor on $X_{0}$ and $D$ be an ample $\boldsymbol{Q}$-Cartier divisor on $X_{0}$. Assume that $g^{*} Q-\left(K_{X}+\Delta+g^{*} D\right)$ is nef and big. Fix one point $x_{0}$ on $X_{0}$.

In the case of $\operatorname{dim} X_{0}=3$, suppose that $g$ is a birational morphism and $D$ has the following numerical criterion:

(1) $D^{3}>\left(\sigma_{3}\right)^{3}$,

(2) $D^{2} \cdot S>\left(\sigma_{2}\right)^{2}$, for all surfaces $S \subset X_{0}$ such that $x_{0} \in S$,

(3) $D \cdot C>\sigma_{1}$, for all curves $C \subset X_{0}$ such that $x_{0} \in C$.

Then if $\sigma_{1}, \sigma_{2}$ and $\sigma_{3}$ satisfy the following conditions:

(1) $\sigma_{3} \geqq \frac{7}{2}$,

(2) $\left(1-\frac{7}{2 \sigma_{3}}\right) \sigma_{1} \geqq 2$, and $\left(1-\frac{7}{2 \sigma_{3}}\right) \sigma_{2} \geqq 2$, $x_{0} \notin \mathrm{Bs}|Q|$.

In the case $\operatorname{dim} X_{0}=2$, suppose $g$ has only connected fibres and $D$ has the following numerical criterion:

(1) $D^{2}>\left(\sigma_{2}\right)^{2}$,

(2) $D \cdot C>\sigma_{1}$, for all curves $C \subset X_{0}$ such that $x_{0} \in C$.

Then if $\sigma_{1}$ and $\sigma_{2}$ satisfy the following conditions:

(1) $\sigma_{2} \geqq 3$,

(2) $\left(1-\frac{3}{\sigma_{2}}\right) \sigma_{1} \geqq 2$,

$x_{0} \notin \mathrm{Bs}|Q|$.

In the case of $\operatorname{dim} X_{0}=1$, suppose that $g$ has only connected fibres and $D$ has the following numerical criterion:

(1) $\operatorname{deg} D>1$.

Then $x_{0} \notin \mathrm{Bs}|Q|$.

Proof of theorems stated in introduction. First we consider Theorem 4. By Base point free theorem [7], we can obtain a normal variety $X_{0}$, a projective morphism $g: X \rightarrow X_{0}$ and an ample Cartier divisor $H$ on $X_{0}$ such that $L \sim g^{*} H$ and $g_{*} \mathcal{O}_{X} \cong \mathcal{O}_{X_{0}}$. Let $Q:=n H$ and $D:=(n-a) H$. If $\operatorname{dim} X_{0}=3$ then the inequalities in Theorem 9 are satisfied with $\sigma_{i}=11 / 2,(i=1,2,3)$. If $\operatorname{dim} X_{0}=2$ then the inequalities in Theorem 9 are satisfied with $\sigma_{\imath}=11 / 2, \quad(i=1,2)$. If $\operatorname{dim} X_{0}=1$ then the inequalities in Theorem 9 are satisfied because $\operatorname{deg} D>11 / 2$. Next we consider Theorem 5 . The pair $(X,(1-\varepsilon) \Delta)$ has only log-terminal singularities for $0<\varepsilon<1$, because $X$ is $\boldsymbol{Q}$-factorial. On the other hand, $a L-$ $\left(K_{X}+(1-\varepsilon) \Delta\right)$ is still ample for $0<\varepsilon \ll 1$. Thus we can reduce this theorem to 
Theorem 4 by replacing $\Delta$ with $(1-\varepsilon) \Delta$. Finally we consider Theorem 6 . Similarly we can obtain a normal 3-fold, a birational morphism $g: X \rightarrow X_{0}$ and an ample $Q$-Cartier divisor $K_{X_{0}}$ such that $K_{X} \sim_{Q} g * K_{X_{0}}$. If $r=1$ then we put $Q:=6 K_{X}$ and $D:=(5-\varepsilon) K_{X}, 0<\varepsilon \ll 1$. Note that $K_{X_{0}}^{3}$ is even. Thus we can put

$$
\sigma_{1}=\frac{24 \sqrt[3]{3}}{5} \text { and } \sigma_{\imath}=\frac{24}{5}, \text { for } i=1,2
$$

in the inequalities of Theorem 9. If $r \geqq 2$ then we put $Q:=m r K_{X_{0}}$ and $D:=$ $(m r-1-\varepsilon) K_{X_{0}}, 0<\varepsilon \ll 1$. Note that $K_{X_{0}}^{3} \in(1 / r) \boldsymbol{Z}$. We can put

$$
\sigma_{1}=\frac{m r-1-\varepsilon}{\sqrt[3]{r}} \text { and } \sigma_{i}=\frac{m r-1-\varepsilon}{r}, \text { for } i=1,2 \text {, }
$$

in the inequalities of Theorem 9 .

Proof of Theorem. We may assume that $X$ has only $\boldsymbol{Q}$-factorial terminal singularities, because by Kawamata [8], there is a normal projective 3 -fold $X^{\prime}$, an effective $\boldsymbol{Q}$-divisor $\Delta^{\prime}$, and a birational morphism $\pi: X^{\prime} \rightarrow X$ which satisfy the following conditions :

(1) $X^{\prime}$ has only $\boldsymbol{Q}$-factorial terminal singularities.

(2) $K_{X^{\prime}}+\Delta^{\prime} \sim_{Q} \pi *\left(K_{X}+\Delta\right)$.

(3) $\left(X^{\prime}, \Delta^{\prime}\right)$ has only log-terminal singularities.

If we replace $X, \Delta, g$ by $X^{\prime}, \Delta^{\prime}, g \circ \pi$ all assumptions of Theorem are satisfied.

Let $B$ be an effective $Q$-divisor on $X$ such that $B \sim_{Q} g^{*} D, Y$ be a smooth projective 3-fold and $f$ be a birational morphism $f: Y \rightarrow X$. We consider a pair $(B, Y, f)$ which satisfy the following conditions :

(1) There is a simple normal crossing divisor $\Sigma E_{\imath}$ on $Y$.

(2) $K_{Y} \sim_{Q} f *\left(K_{X}+\Delta\right)+\sum e_{\imath} E_{\imath}, e_{\imath}>-1$.

(3) $f * B=\sum b_{i} E_{\imath}, b_{i} \geqq 0$.

(4) There is an ample $Q$-Cartier divisor $A$ on $Y$ such that $f *\left(g * Q-\left(K_{X}+\right.\right.$ $\left.\left.\Delta+g^{*} D\right)\right) \sim_{Q} A+\sum p_{i} E_{\imath}, 0<p_{i} \ll 1$ and $e_{i}+1-p_{i}>0$.

We define

$$
c:=\min _{\imath}\left\{\frac{e_{i}+1-p_{i}}{b_{i}} \mid x_{0} \in g \circ f\left(E_{\imath}\right), b_{i}>0\right\} .
$$

By changing indices, if necessary, we may assume that the minimum $c$ attained only at a unique index $i=0$. We obtain the following lemma.

LEMMA 5. (1) If $c b_{i}-e_{i}+p_{i}<0$, then $E_{\imath}$ is a f-exceptional divisor.

(2) If $c b_{i}-e_{i}+p_{i}>1$, then $x_{0} \notin g \circ f\left(E_{\imath}\right)$.

Proof. (1) If $c b_{i}-e_{i}+p_{i}<0$, then $e_{2}>c b_{i}+p_{i}$. By $b_{i} \geqq 0, p_{i}>0$, and $c>0$, we obtain $e_{\imath}>0$.

(2) If $b_{i}>0$ and $x_{0} \in g \circ f\left(E_{\imath}\right)$, then $c \leqq\left(e_{i}+1-p_{i}\right) / b_{i}$. From this inequality, we obtain $c b_{i}-e_{i}+p_{i} \leqq 1$. If $b_{i}=0$ and $c b_{i}-e_{i}+p_{i}>1$, then $e_{i}<-1+p_{i}$. But 
this inequality contradicts $e_{i}+1-p_{i}>0$.

We go back the proof of Theorem 9. Let $R:=\Sigma\left(c b_{i}-e_{\imath}+p_{i}\right) E_{\imath}$. Then we can write $[R]=E_{0}+N-P$, where $E_{0}, N$ and $P$ are effective Cartier divisors which satisfy the following conditions:

(1) $E_{0}, N$ and $P$ have no common components.

(2) $P$ is a composite of the $f$-exceptional divisors.

(3) $\quad N$ is a composite of the divisors $E_{\imath}$ such that $x_{0} \notin g \circ f\left(E_{\imath}\right)$.

We define

$$
T:=(g \circ f) * Q+P-E_{0}-N-K_{Y}-\langle R\rangle .
$$

Then

$$
T \sim_{Q}(1-c)(g \circ f)^{*} D+A
$$

by

$$
(g \circ f) * Q+P-N-E_{0} \sim_{Q} K_{Y}+(1-c)(g \circ f) * D+A+\Sigma\left\langle c b_{i}-e_{i}+p_{i}\right\rangle E_{\imath} .
$$

Proposition 3. For a suntable pair $(B, Y, f)$,

(1) $c<\frac{7}{2 \sigma_{3}}$, if $\operatorname{dim} X_{0}=3$.

(2) $c<\frac{3}{\sigma_{2}}$, if $\operatorname{dim} X_{0}=2$.

(3) $c<\frac{1}{\operatorname{deg} D}$, if $\operatorname{dim} X_{0}=1$.

We will prove Proposition 3 in section 6 . By the conditions of theorem:

(1) $\sigma_{3} \geqq \frac{7}{2},\left(\operatorname{dim} X_{0}=3\right)$.

(2) $\sigma_{2} \geqq 3,\left(\operatorname{dim} X_{0}=2\right)$.

(3) $\operatorname{deg} D>1,\left(\operatorname{dim} X_{0}=1\right)$.

and Proposition 3, we get $1-c>0$. Thus $T$ is nef and big. $\operatorname{Supp}\langle T\rangle=\operatorname{Supp}\langle R\rangle$ is a simple normal crossing divisor. Therefore by Kawamata-Viehweg Vanishing Theorem,

$$
H^{1}\left(Y, K_{Y}+\lceil T\urcorner\right) \cong H^{1}\left(Y,(g \circ f) * Q+P-N-E_{0}\right)=0 .
$$

Hence the restriction map

$$
H^{0}(Y,(g \circ f) * Q+P-N) \longrightarrow H^{0}\left(E_{0},\left.((g \circ f) * Q+P-N)\right|_{E_{0}}\right)
$$

is surjective.

LEMMA 6. In the following diagram, 


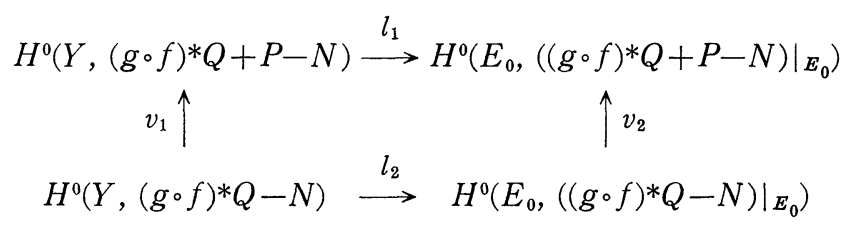

the bottom horizontal map $l_{2}$ is surjective.

Proof. For the proof of this lemma, we need the following lemma.

Lemma 7 ([7] Th. 1-5-2). Let $X$ be a smooth projective variety, $Y$ be a normal projective variety, and $f: X \rightarrow Y$ be a birational morphism. Let $Q$ be $a$ $f$-exceptional divisor on $X$. Then $f_{*} O_{Q}(Q)=0$.

$P$ and $N$ have no common components. So by Lemma $7, f_{*} \Theta_{P}(P-N)=0$. Hence $v_{1}$ is isomorphism. Moreover $v_{2}$ is injective and $l_{1}$ is surjective. By the commutatibity of this diagram, $l_{2}$ is surjective.

Proposition 4. There is a section $t \in H^{0}\left(E_{0},\left.((g \circ f) * Q-N)\right|_{\boldsymbol{E}_{0}}\right)$ which dose not vanish at $(g \circ f)^{-1}\left(x_{0}\right)$.

By Proposition 4 and Lemma 6, there is a section $s \in H^{\circ}(Y,(g \circ f) * Q-N)$ which doesn't vanish at $(g \circ f)^{-1}\left(x_{0}\right)$. Because $x_{0} \notin g \circ f(N)$, we can deduce $x_{0} \notin$ $\operatorname{Bs}|Q|$.

For completing the proof of theorem, it is enough to show Proposition 3 and Proposition 4. We will show Proposition 4 at the section 5 and Proposition 3 at the section 6 .

\section{The existence of desirable section}

\section{Proof of Proposition 4.}

The Case $\operatorname{dim} g \circ f\left(E_{0}\right)=0$.

Since $x_{0} \notin g \circ f(N), \mathcal{O}_{E_{0}}((g \circ f) * Q-N) \cong \mathcal{O}_{E_{0}}$. Thus the assertion of Proposition 4 follows immediately.

The Case $\operatorname{dim} g \circ f\left(E_{0}\right)=1$.

Let $C:=g \circ f\left(E_{0}\right)$. Take a stein factorization $E_{0} \stackrel{\rho}{\rightarrow} C^{\prime} \stackrel{\nu}{\rightarrow} C$ of $E_{0} \stackrel{g \circ f}{\rightarrow} C$. Then $C^{\prime}$ is a smooth curve and $\rho$ is a flat morphism. Fix one point $x_{1} \in \nu^{-1}\left(x_{0}\right)$. Let $Z$ be a scheme theoretic inverse of $x_{1}$.

LEMMA 8. The restriction map

$$
H^{0}\left(E_{0},\left.((g \circ f) * Q-N)\right|_{E_{0}}\right) \stackrel{i}{\longrightarrow} H^{0}\left(Z,\left.((g \circ f) * Q-N)\right|_{Z}\right)
$$

is nonzero map. 
Since $H^{0}\left(C^{\prime}, \nu^{*} Q\right) \cong H^{0}\left(E_{0},\left.((g \circ f) * Q)\right|_{E_{0}}\right)$, the section $s \in H^{0}\left(E_{0},\left.((g \circ f) * Q)\right|_{E_{0}}\right)$ which vanish at some point of $(g \circ f)^{-1}\left(x_{1}\right)$ is identically zero on $Z$. Thus, if Lemma 8 is valid, there is a section $t \in H^{0}\left(E_{0},\left.((g \circ f) * Q-N)\right|_{E_{0}}\right)$ which doesn't vanish some point of $(g \circ f)^{-1}\left(x_{0}\right)$.

Proof of Lemma 8. First we prove the following claim.

Claim 5. (1) $H^{1}\left(E_{0},\left.((g \circ f) * Q+P-N)\right|_{E_{0}}-Z\right)=0$.

(2) $H^{0}\left(Z,\left.((g \circ f) * Q+P-N)\right|_{z}\right) \neq 0$.

Proof. (1) Let $\Delta_{E_{0}}:=\sum\left\langle c b_{i}-e_{i}+p_{i}\right\rangle\left(\left.E_{\imath}\right|_{E_{0}}\right)$. We define

$$
U:=\left.((g \circ f) * Q+P-N)\right|_{E_{0}}-Z-K_{E_{0}}-\Delta_{E_{0}} .
$$

Then

because

$$
\begin{aligned}
U & \sim_{Q}(1-c)(\rho \circ \nu) *\left(\left.D\right|_{C}\right)+\left.A\right|_{E_{0}}-Z, \\
& \sim_{Q} \rho^{*} \mathcal{O}_{C^{\prime}}\left((1-c) \nu^{*}\left(\left.D\right|_{C}\right)-x_{1}\right)+\left.A\right|_{E_{0}},
\end{aligned}
$$

$$
\left.((g \circ f) * Q+P-N)\right|_{E_{0}} \sim_{Q} K_{E_{0}}+\Delta_{E_{0}}+\left.(1-c)((g \circ f) * D)\right|_{E_{0}}+\left.A\right|_{E_{0}},
$$

by the equation $(1)$ and $\left.((g \circ f) * D)\right|_{E_{0}}=(\rho \circ \nu) *\left(\left.D\right|_{C}\right)$. We obtain

$$
\operatorname{deg} \rho^{*} \mathcal{O}_{C}\left((1-c) \nu^{*}\left(\left.D\right|_{C}\right)-x_{1}\right)>(1-c) \sigma_{1}-1 \text {. }
$$

By Proposition 3,

$$
c<\frac{7}{2 \sigma_{3}}, \quad\left(\operatorname{dim} X_{0}=3\right), \quad \text { and } \quad c<\frac{3}{\sigma_{2}}, \quad\left(\operatorname{dim} X_{0}=2\right) .
$$

Thus

$$
(1-c) \sigma_{1}>\left(1-\frac{7}{2 \sigma_{3}}\right) \sigma_{1} \geqq 2, \quad\left(\operatorname{dim} X_{0}=3\right),
$$

and

$$
(1-c) \sigma_{1}>\left(1-\frac{3}{\sigma_{2}}\right) \sigma_{1} \geqq 2, \quad\left(\operatorname{dim} X_{0}=2\right),
$$

by the assumption of theorem. Hence $U$ is ample. On the other hand $\operatorname{Supp}\langle U\rangle$ $=\operatorname{Supp}\left\langle\Delta_{E_{0}}\right\rangle$ is simple normal crossing divisor. Therefore by KawamataViehweg Vanishing Theorem

$$
H^{1}\left(E_{0}, K_{E_{0}}+\lceil U\urcorner\right) \cong H^{1}\left(E_{0},\left.((g \circ f) * Q+P-N)\right|_{E_{0}}-Z\right)=0 .
$$

(2) Since $x \notin g \circ f(N)$, there is a point $x^{\prime}$ on $C^{\prime}$ such that $x^{\prime} \notin g \circ f(N)$. For $Z^{\prime}:=\rho^{-1}\left(x^{\prime}\right), H^{0}\left(Z^{\prime},\left.((g \circ f) * Q+P-N)\right|_{Z^{\prime}}\right)=H^{0}\left(Z^{\prime},\left.P\right|_{Z^{\prime}}\right) \neq 0$, Because $\rho$ is flat, $H^{0}\left(Z,\left.((g \circ f) * Q+P-N)\right|_{Z}\right) \neq 0$ by semicontinuity.

We go back the proof of Lemma 8. Consider the following diagram: 


$$
\begin{gathered}
H^{0}\left(E_{0},\left.((g \circ f) * Q+P-N)\right|_{E_{0}}\right) \stackrel{l_{1}}{\longrightarrow} H^{0}\left(Z,\left.((g \circ f) * Q+P-N)\right|_{Z}\right) \\
v_{1} \uparrow \\
H^{0}\left(E_{0},\left.((g \circ f) * Q-N)\right|_{E_{0}}\right) \stackrel{\uparrow v_{2}}{\longrightarrow} H^{0}\left(Z,\left.\left((g \circ f)^{*} Q-N\right)\right|_{Z}\right) .
\end{gathered}
$$

From Claim 5, $l_{1}$ is surjection and $v_{2}$ is nonzero map and injection. On the other hand $v_{1}$ is isomorphism. Thus $l_{2}$ is surjection. Moreover $H^{0}(Z,((g \circ f) * Q$ $\left.-N)\left.\right|_{Z}\right) \neq 0$, because $\mathcal{O}_{Z}((g \circ f) * Q-N) \cong \mathcal{O}_{Z}$. Therefore the assertion of Lemma 8 follows.

The Case $\operatorname{dim} g \circ f\left(E_{0}\right)=2$

Let $S_{1}:=g \circ f\left(E_{0}\right)$. We define that $P_{1}$ is composition of the components $E_{2}$ of $P$ such that $x_{0} \in g \circ f\left(E_{\imath}\right)$. Let $P_{2}:=P-P_{1}$. We denote by $f^{\prime}$ (resp. $\left.g^{\prime}\right)$ the restriction morphism $\left.f\right|_{E_{0}}$ (resp. $\left.\left.g\right|_{f\left(E_{0}\right)}\right)$.

LEMMA 9. (1) $S_{1}$ is normal in a neighborhood of $x_{0}$.

(2) $\left.P_{1}\right|_{E_{0}}$ is $f^{\prime}$-exceptional.

Proof. Consider the following diagram:

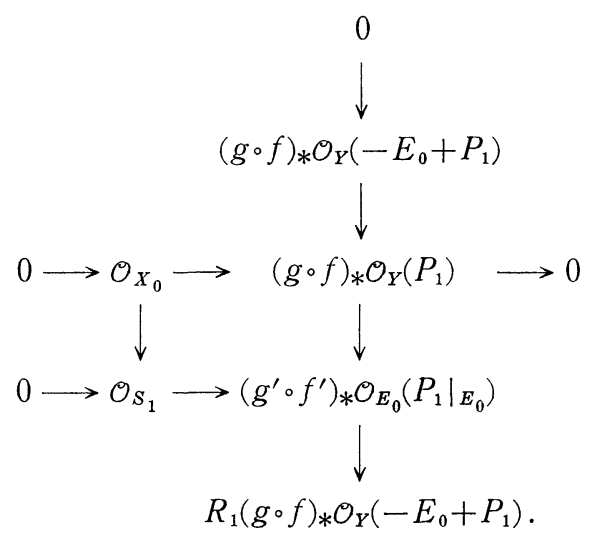

We define

$$
T^{\prime}:=-E_{0}+P_{1}-K_{Y}-\Sigma\left\langle c b_{i}-b_{i}+p_{i}\right\rangle E_{\imath} .
$$

Then

$$
T^{\prime} \sim_{Q}-(g \circ f) * Q-P_{2}+N+A+(1-c)(g \circ f) * D,
$$

by the equation (1). For a curve such that $C \subset(g \circ f)^{-1}\left(x_{0}\right), P_{2} \cdot C=N \cdot C=0$, because $\operatorname{Supp}\left(P_{2}+N\right) \cap(g \circ f)^{-1}\left(x_{0}\right)=\emptyset$. Thus $T^{\prime}$ is $g \circ f$-ample in a some neighborhood $V$ of $x_{0}$. Supp $\left\langle T^{\prime}\right\rangle$ is a simple normal crossing divisor. Therefore, by Kawamata-Viehweg Vanishing Theorem,

$$
R^{1}(g \circ f)_{*} \mathcal{O}_{Y}\left(K_{Y}+\left.\left.\left\lceil T^{\prime} 7\right)\right|_{V} \cong R^{1}(g \circ f)_{*} \mathcal{O}_{Y}\left(-E_{0}+P_{1}\right)\right|_{V}=0 .\right.
$$


Hence $\left.\left.\left(g^{\prime} \circ f^{\prime}\right)_{*} \mathcal{O}_{E_{0}}\left(\left.P_{1}\right|_{E_{0}}\right)\right|_{V} \cong \mathcal{O}_{S_{1}}\right|_{V}$. The assertions of Lemma follows by this isomorphism.

We go back the proof of Proposition 4. Since $S_{1}$ is normal in a some neighborhood $V$ of $x_{0}$, by Lemma 9, there is an open neighborhood $V^{\prime}$ of $x_{0}$ such that $g^{\prime} \circ f^{\prime}$ is an isomorphism over $V^{\prime} \backslash\left\{x_{0}\right\}$. Let $Z:=\left(g^{\prime} \circ f^{\prime}\right)^{-1}\left(x_{0}\right)$. For $Z$, we define $p: E_{0} \rightarrow S_{0}$ be a contraction morphism of $Z$, and $q: S_{0} \rightarrow S_{1}$ be an induced morphism. Then $q$ is isomorphism over $V^{\prime}$. Let $\Delta_{E_{0}}:=\Sigma\left\langle c b_{i}-e_{i}+p_{i}\right\rangle\left(\left.E_{\imath}\right|_{E_{0}}\right)$. Take a relative $\log$ minimal model $\left(S, \Delta_{S}\right)$ of $\left(E_{0}, \Delta_{E_{0}}\right)$ over $S_{1}$.

LEMMA $10 . \quad S \cong S_{0}$.

Proof. Let

$$
E_{0} \stackrel{\rho}{\longrightarrow} S \stackrel{\nu}{\longrightarrow} S_{0}
$$

First we show $\rho_{*}\left(\left.P_{1}\right|_{E_{0}}\right)=0$. Assume the contrary. Because $\left.P_{1}\right|_{E_{0}}$ is $g^{\prime} \circ f^{\prime}$ exceptional and $\left(S_{0}, x_{0}\right)$ is a normal point, there is a curve $C \subset \operatorname{Supp}\left(\rho_{*}\left(\left.P_{1}\right|_{E_{0}}\right)\right)$ such that $\rho_{*}\left(\left.P_{1}\right|_{E_{0}}\right) \cdot C<0$. Since $\rho_{*}\left(K_{E_{0}}+\Delta_{E_{0}}\right)=K_{S}+\Delta_{S}$ and

$$
K_{E_{0}}+\left.(1-c)((g \circ f) * D)\right|_{E_{0}}+\left.A\right|_{E_{0}}+\left.\Delta_{E_{0}} \sim_{Q}((g \circ f) * Q+P-N)\right|_{E_{0}}
$$

by the equation (1), we obtain

$$
K_{S}+\Delta_{S} \sim_{Q}(q \circ \nu) * Q+\rho_{*}\left(\left.\left(P_{1}+P_{2}-N\right)\right|_{E_{0}}\right)-\rho_{*}\left(\left.A\right|_{E_{0}}\right)-(1-c)(q \circ \nu) * D .
$$

Since $\operatorname{Supp}\left(P_{2}+N\right) \cap(g \circ f)^{-1}\left(x_{0}\right)=\emptyset$,

$$
\left(K_{S}+\Delta_{S}\right) \cdot C=-\rho_{*}\left(\left.A\right|_{E_{0}}\right) \cdot C+\rho_{*}\left(\left.P_{1}\right|_{E_{0}}\right) \cdot C .
$$

Because $\rho$ is a birational morphism of surfaces, ample divisor's push-forward is also ample. Thus $\left(K_{S}+\Delta_{S}\right) \cdot C<0$, which contradicts with the assumption that $\left(S, \Delta_{S}\right)$ is log minimal model. Hence $\rho_{*}\left(\left.P_{1}\right|_{E_{0}}\right)=0$. Next we show $\nu^{-1}\left(x_{0}\right)$ is one point. Assume the contrary. Take a curve $C \subset \nu^{-1}\left(x_{0}\right)$. Then

$$
\left(K_{S}+\Delta_{S}\right) \cdot C=-\rho_{*}\left(\left.A\right|_{E_{0}}\right) \cdot C<0,
$$

which is contradiction. Therefore $\nu$ is isomorphism.

We go back the proof of Proposition 4. For completing the proof of Proposition 4, we need the following lemma:

LEMMA 11. $\left.\left(S, \Delta_{S}\right),\left.p_{*}((g \circ f) * Q+P-N)\right|_{E_{0}}\right)$ are satisfy the assumptions of Theorem 7.

By this lemma, we can choose a section $s \in H^{0}\left(S,\left.p_{*}((g \circ f) * Q+P-N)\right|_{E_{0}}\right)$ such that $s\left(x_{0}\right) \neq 0$. Because $P_{1}$ is $p$-exceptional, 


$$
\left.p^{*}\left(\left.p_{*}((g \circ f) * Q+P-N)\right|_{E_{0}}\right) \sim\left((g \circ f) * Q+P_{2}-N\right)\right|_{E_{0}} .
$$

So there is a setion $\left.\left.s^{\prime} \in H^{0}\left(E_{0},(g \circ f)^{*} Q+P_{2}-N\right)\right|_{E_{0}}\right)$ which does not vanish at some point of $E_{0} \cap(g \circ f)^{-1}\left(x_{0}\right)$. Then consider the following diagram.

$$
\begin{array}{ccc}
H^{0}\left(Y, \mathcal{O}_{Y}((g \circ f) * Q+P-N)\right) \stackrel{l_{1}}{\longrightarrow} & H^{0}\left(E_{0}, \mathcal{O}_{E_{0}}\left(\left.((g \circ f) * Q+P-N)\right|_{E_{0}}\right)\right) \\
v_{1} \uparrow v_{21} & \\
& H^{0}\left(E_{0}, \mathcal{O}_{E_{0}}\left(\left.\left((g \circ f) * Q+P_{2}-N\right)\right|_{E_{0}}\right)\right) \\
H^{0}\left(Y, \mathcal{O}_{Y}((g \circ f) * Q-N)\right) & \stackrel{l_{2}}{\longrightarrow} & H^{0}\left(E_{0}, \mathcal{O}_{E_{0}}\left(((g \circ f) * Q-N)_{E_{0}}\right)\right)
\end{array}
$$

Already we show that $v_{1}$ is isomorphism and $l_{1}, l_{2}$ are surjective. Moreover $v_{21}$, $v_{22}$ are injective, we can deduce $v_{21}, v_{22}$ are isomorphism. Then the section $v_{22}^{-1}\left(s^{\prime}\right)$ has the desirable property.

Proof of Lemma 11. First we show the following claim.

Claim 6. Let $M:=p_{*}\left(\left.((g \circ f) * Q+P-N)\right|_{E_{0}}\right)-\left(K_{S}+\Delta_{S}\right)$. Then $M$ satisfy the following conditions:

(1) $M$ is nef and big,

(2) $M^{2}>4$,

(3) $M \cdot C>2$ for all curves $C$ such that $q^{-1}\left(x_{0}\right) \in C$.

Proof. By the equation (1),

$$
\left.\left.((g \circ f) * Q+P-N)\right|_{E_{0}}\right) \sim_{Q} K_{E_{0}}+\left.(1-c)((g \circ f) * D)\right|_{E_{0}}+\left.A\right|_{E_{0}}+\Delta_{E_{0}} .
$$

Since $\left(S, \Delta_{S}\right)$ is a log minimal model of $\left(E_{0}, \Delta_{E_{0}}\right), p_{*}\left(K_{E_{0}}+\Delta_{E_{0}}\right)=K_{S}+\Delta_{S}$. Because $\left.((g \circ f) * D)\right|_{E_{0}}=\left(g^{\prime} \circ f^{\prime}\right) *\left(\left.D\right|_{S_{1}}\right)$ and $g^{\prime} \circ f^{\prime}=q^{\circ} p$, we obtain $\left.p_{*}((g \circ f) * D)\right|_{E_{0}}=$ $q^{*}\left(\left.D\right|_{S_{1}}\right)$. Therefore

$$
\left.M \sim_{Q}(1-c) q^{*} D\right|_{S_{1}}+\left.p_{*} A\right|_{E_{0}} .
$$

Then $M$ is nef and big because $\left.D\right|_{S_{1}}$ is ample, $p$ is birational and $\left.A\right|_{E_{0}}$ is ample. Furthermore

and

$$
M^{2}=\left(\left.(1-c) q^{*} D\right|_{S_{1}}+\left.p_{*} A\right|_{E_{0}}\right)^{2}>(1-c)^{2}\left(q^{*}\left(\left.D\right|_{S_{1}}\right)\right)^{2},
$$

$$
M \cdot C=\left(\left.(1-c) q^{*} D\right|_{s_{1}}+\left.p_{*} A\right|_{E_{0}}\right) \cdot C>\left((1-c) q^{*}\left(\left.D\right|_{S_{1}}\right)\right) \cdot C,
$$

where $C$ is a curve such that $q^{-1}\left(x_{0}\right) \in C$. By the assumption of theorem,

$$
(1-c)^{2}\left(q^{*}\left(\left.D\right|_{S_{1}}\right)\right)^{2}=(1-c)^{2}\left(\left.D\right|_{S_{1}}\right)^{2}=(1-c)^{2} D^{2} \cdot S_{1}>\left((1-c) \sigma_{2}\right)^{2},
$$

and 


$$
(1-c) q^{*}\left(\left.D\right|_{S_{1}}\right) \cdot C=\left.(1-c) D\right|_{S_{1}} \cdot q(C)=(1-c) D \cdot q(C)>(1-c) \sigma_{1},
$$

because $q$ is an isomorphism in a neighborhood of $\left\{x_{0}\right\}$. By Proposition $3, c<$ $7 /\left(2 \sigma_{3}\right)$. Thus $1-c>1-7 /\left(2 \sigma_{3}\right)$. Then, again by the assumption of theorem

$$
\left(1-\frac{7}{2 \sigma_{3}}\right) \sigma_{2} \geqq 2, \quad \text { and } \quad\left(1-\frac{7}{2 \sigma_{3}}\right) \sigma_{1} \geqq 2 .
$$

Hence we can deduce the assertion of claim.

We go back the proof of Lemma 11. By Claim 6, we only have to show that $p_{*}\left(\left.((g \circ f) * Q+P-N)\right|_{E_{0}}\right)$ is Cartier divisor.

$$
p_{*}\left(\left.\left((g \circ f)^{*} Q+P-N\right)\right|_{E_{0}}\right)=p_{*}\left(\left(g^{\prime} \circ f^{\prime}\right)^{*}\left(\left.Q\right|_{S_{1}}\right)+\left.P_{1}\right|_{E_{0}}+\left.P_{2}\right|_{E_{0}}-\left.N\right|_{E_{0}}\right) .
$$

Since $\left.P_{1}\right|_{E_{0}}$ is $p$-excetional and $g^{\prime} \circ f^{\prime}=q \circ p$,

$$
\begin{aligned}
& p_{*}\left(\left(g^{\prime} \circ f^{\prime}\right) *\left(\left.Q\right|_{S_{1}}\right)+\left.P_{1}\right|_{E_{0}}+\left.P_{2}\right|_{E_{0}}-\left.N\right|_{E_{0}}\right) \\
& \quad=p_{*}(q \circ p)^{*}\left(\left.Q\right|_{S_{1}}\right)+p_{*}\left(\left.P_{2}\right|_{E_{0}}\right)-p_{*}\left(\left.N\right|_{E_{0}}\right) \\
& \quad=\left.q_{*} Q\right|_{S_{1}}+p_{*}\left(\left.P_{2}\right|_{E_{0}}\right)-p_{*}\left(\left.N\right|_{E_{0}}\right) .
\end{aligned}
$$

Then $p_{*}\left(\left.P_{2}\right|_{E_{0}}\right)-p_{*}\left(\left.N\right|_{E_{0}}\right)$ is Cartier divisor because $p$ is isomorphism over $S \backslash\left\{q^{-1}\left(x_{0}\right)\right\}$. Therefore $p_{*}\left(\left.((g \circ f) * Q+P-N)\right|_{E_{0}}\right)$ is Cartier divisor. This completes the proof of Lemma 11.

Now we have completed the proof of Proposition 4.

\section{Estimation of $c$}

Proof of Proposition 3.

(1) Let $B$ be an effective $\boldsymbol{Q}$-divisor on $X, Y$ be a smooth 3 -fold and $f$ be a birational morphism $f: Y \rightarrow X$. We will construct the pair $(B, Y, f)$ which satisfy the following conditions:

(1) There is a simple normal crossing divisor $\Sigma E_{\imath}$ on $Y$.

(2) $K_{Y} \sim_{Q} f *\left(K_{X}+\Delta\right)+\sum e_{i} E_{\imath}, e_{\imath}>-1$.

(3) $f * B=\sum b_{i} E_{\imath}$.

(4) There is an ample $\boldsymbol{Q}$-divisor $A$ on $Y$ such that $f *\left(g * Q-\left(K_{X}+\Delta+g * D\right)\right)$ $\sim_{Q} A+\sum p_{i} E_{\imath}, 0<p_{i} \ll 1$ and $e_{i}+1-p_{i}>0$.

We construct these objects dividing into three cases:

CASE 1.1. There is a smooth point in $g^{-1}\left(x_{0}\right)$.

CASE 1.2. $g^{-1}\left(x_{0}\right)$ is one point, and $\left(X, x_{1}\right)$ is terminal singularity of index one, where $x_{1}:=g^{-1}\left(x_{0}\right)$. 
CASE 1.3. $g^{-1}\left(x_{0}\right)$ is one point, and $\left(X, x_{1}\right)$ is terminal singularity of index $r,(r \geqq 2)$, where $x_{1}:=g^{-1}\left(x_{0}\right)$.

If there is no smooth points in $g^{-1}\left(x_{0}\right), g^{-1}\left(x_{0}\right)$ is one point, because three dimensional terminal singularity is isolated singularity.

CASE 1.1. Let $x_{1} \in g^{-1}\left(x_{0}\right)$ be a smooth point. By Lemma 1, we can choose an effective $\boldsymbol{Q}$-divisor $B$ on $X$ such that $\nu_{x_{1}}(B)>\sigma_{3} /(1-\boldsymbol{\sigma})$ and $B \sim_{\boldsymbol{Q}} g^{*} D$. Let $h: X^{\prime} \rightarrow X$ be the blowing up with center $x_{1}$. We take a resolution of singularities $f_{0}: Y^{\prime} \rightarrow X^{\prime}$ such that the union of the exceptional locus and the proper transform of $\Delta+B$ by $h \circ f_{0}$ is a divisor with only simple normal crossings. By Kodaira's Lemma, we can take an effective divisor $D^{\prime}$ such that $\left(h \circ f_{0}\right) *(g * Q-$ $\left.\left(K_{X}+\Delta+g^{*} D\right)\right)-\delta D^{\prime}$ is ample for $0<\delta<1$. Taking a succession of blowing-ups with nonsingular center, we can find a smooth variety $Y$ and a birational morphism $f_{1}: Y \rightarrow Y^{\prime}$ which satisfies the following properties:

(1) the union of the $h \circ f_{0} \circ f_{1}$-exceptional locus, the proper transform of $\Delta+B$ by $h \circ f_{0} \circ f_{1}$ and the proper transform of $D^{\prime}$ by $f_{1}$ is a divisor with only simple normal crossings.

(2) $\left(h \circ f_{0} \circ f_{1}\right) *\left(g * Q-\left(K_{X}+\Delta+g^{*} D\right)\right)-\delta f_{1}^{*} D^{\prime}-\Sigma d_{i}^{\prime} E_{\imath}$ is ample for $0<d_{i} \ll 1$, where $\Sigma d_{i}^{\prime} E_{\imath}$ is an exceptional divisor.

If we write $K_{Y} \sim_{Q}\left(h \circ f_{0} \circ f_{1}\right) *\left(K_{X}+\Delta\right)+\sum e_{i} E_{\imath}$, then $e_{\imath}>-1$ because $(X, \Delta)$ has only log-terminal singularities. If we take $\delta$ and $d_{\imath}^{\prime}$ small enough, we can obtain $e_{i}+1-p_{i}>0$ by the Logarithmic Ramification Formula. Thus the pair $\left(B, Y, f:=h \circ f_{0} \circ f_{1}\right)$ satisfies the above four conditions. Suppose $F$ be the exceptional divisor of $h$. Let $E_{1}$ be the proper transform of $F$ by $f_{0} \circ f_{1}$. Then $b_{1}>\sigma_{3} /(1-\sigma), e_{1} \leqq 2$. Thus

$$
c \leqq \frac{e_{1}+1-p_{1}}{b_{1}}<\frac{e_{1}+1}{b_{1}}<\frac{3(1-\sigma)}{\sigma_{3}} .
$$

CASE 1.2. Let $x_{1}:=g^{-1}\left(x_{0}\right)$. Since $x_{1}$ is a cDV singularity, mult $x_{x_{1}} X=2$. By Lemma 1, we can choose an effective $\boldsymbol{Q}$-divisor $B$ on $X$ such that $\nu_{x_{1}}(B)>$ $\sigma_{3} / \sqrt[3]{2}(1-\sigma)$ and $B \sim_{Q} g^{*} D$. Take the blowing up $h: X^{\prime} \rightarrow X$ with center $x_{1}$. Then $X^{\prime}$ is a normal 3 -fold which has only Gorenstein singularity and there is a reduced divisor $F$ on $X^{\prime}$ such that $K_{X^{\prime}} \sim h^{*} K_{X}+F$. Choose a smooth variety $Y$ and a birational morphism $f^{\prime}: Y \rightarrow X^{\prime}$ as in Case 1.1 such that the pair $\left(B, Y, f:=h \circ f^{\prime}\right)$ satisfies the above four conditions. Let $E_{1}$ be the proper transform of $F$ by $f$. Then $b_{1}>\sigma_{3} / \sqrt[3]{2}(1-\sigma), e_{1} \leqq 1$. Thus

$$
c \leqq \frac{e_{1}+1-p_{1}}{b_{1}}<\frac{e_{1}+1}{b_{1}}<\frac{2 \sqrt[3]{2}(1-\sigma)}{\sigma_{3}} .
$$

CASE 1.3. By Kawamata [8], we can choose a normal variety $X_{2}$ and a birational morphism $h: X^{\prime} \rightarrow X$ which satisfy the following conditions : 
(1) $h$ is an isomorphism over $X \backslash\left\{x_{1}\right\}$.

(2) $K_{X^{\prime}} \sim_{Q} h^{*} K_{X}+(1 / r) G+$ (other components), where $G$ is a reduced component of the exceptional divisor of $h$ and $r$ is an index of $\left(X, x_{1}\right)$.

Take a smooth point $x_{2} \in \operatorname{Supp} G$ of $X^{\prime}$. By Lemma 1 , we can choose an effective $\boldsymbol{Q}$-divisor $B^{\prime}$ on $X^{\prime}$ such that $\nu_{x_{2}}\left(B^{\prime}\right)>\sigma_{3} /(1-\sigma)$ and $B^{\prime} \sim_{\boldsymbol{Q}}(g \circ h)^{*} D$. We put $B:=h_{*} B^{\prime}$. After take the blowing-up $h^{\prime}: X^{\prime \prime} \rightarrow X^{\prime}$ with center $x_{2}$, we select a smooth variety $Y$ and a birational morphism $f^{\prime}$ as in Case 1.1 such that the pair $\left(B, Y, f:=h \circ h^{\prime} \circ f^{\prime}\right)$ satisfies the above four conditions. Suppose $F$ the exceptional divisor of $h^{\prime}$. Let $E_{1}$ be the proper transform of $F$ by $f^{\prime}$. Then $b_{1}>\sigma_{3} /(1-\sigma), e_{1} \leqq 2+1 / r \leqq 5 / 2$. Thus

$$
c \leqq \frac{e_{1}+1-p_{1}}{b_{1}}<\frac{e_{1}+1}{b_{1}}<\frac{7(1-\sigma)}{2 \sigma_{3}} .
$$

Now we complete the proof of Proposition 3 of (1).

(2) We will construct normal varieties $X^{\prime}, X_{1}$, and a reduced divisor $F$ on $X^{\prime}$ which satisfy the following conditions:

(1) There is birational morphisms $h: X^{\prime} \rightarrow X$, and $\bar{h}: X_{1} \rightarrow X_{0}$.

(2) There is a commutative diagram:

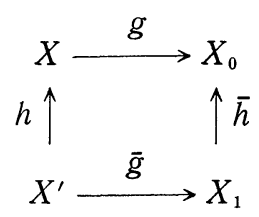

(3) $\vec{h}(F)$ is one point $x_{1}$, where $\left(X_{1}, x_{1}\right)$ is a smooth point and $x_{1} \in \vec{h}^{-1}\left(x_{0}\right)$.

(4) $K_{X^{\prime}} \sim_{Q} h^{*} K_{X}+a F+$ (other components), $(a \leqq 2)$.

First we show if we construct these objects, the assertion of proposition follows. By Lemma 1, we can choose an effective $\boldsymbol{Q}$-divisor $B^{\prime}$ such that $B^{\prime} \sim_{\boldsymbol{Q}} \bar{h}^{*} D$ and $\nu_{x_{1}}\left(B^{\prime}\right)>\sigma_{2} /(1-\sigma),(0<\sigma \ll 1)$. Let $B:=h_{*} \bar{g}^{*} B^{\prime}$. Take a smooth variety $Y$ and a birational morphism $f^{\prime}: Y \rightarrow X^{\prime}$ as in Case 1.1 such that the pair $(B, Y, f:=$ $\left.g \circ h \circ f^{\prime}\right)$ satisfies the four conditions in (1). Let $E_{1}$ be the proper transform of $F$. By the definition of $c$,

$$
c \leqq \frac{e_{1}+1-p_{1}}{b_{1}} .
$$

By construction of $F$ and $B, e_{1} \leqq 2$ and $b_{1}>\sigma_{2} /(1-\sigma)$. Thus

$$
\begin{aligned}
c & \leqq \frac{\left(2+1-p_{1}\right)(1-\sigma)}{\sigma_{2}} \\
& <\frac{3}{\sigma_{2}} .
\end{aligned}
$$


We construct these objects dividing into six cases.

CASE 2.1. $\left(X_{0}, x_{0}\right)$ is smooth point, and $\operatorname{dim} g^{-1}\left(x_{0}\right)=2$.

CASE 2.2. $\left(X_{0}, x_{0}\right)$ is smooth point, $\operatorname{dim} g^{-1}\left(x_{0}\right)=1$, and there is a singular point of $X$ in $g^{-1}\left(x_{0}\right)$.

CASE 2.3. $\left(X_{0}, x_{0}\right)$ is smooth point, $\operatorname{dim} g^{-1}\left(x_{0}\right)=1$, and there is no smooth points of $X$ in $g^{-1}\left(x_{0}\right)$.

CASE 2.4. $\left(X_{0}, x_{0}\right)$ is singular point, and $\operatorname{dim} g^{-1}\left(x_{0}\right)=2$.

CASE 2.5. $\left(X_{0}, x_{0}\right)$ is singular point, $\operatorname{dim} g^{-1}\left(x_{0}\right)=1$, and there is a singular point of $X$ in $g^{-1}\left(x_{0}\right)$.

CASE 2.6. $\left(X_{0}, x_{0}\right)$ is singular point, $\operatorname{dim} g^{-1}\left(x_{0}\right)=1$, and there is no singular points of $X$ in $g^{-1}\left(x_{0}\right)$.

CONSTRUCTION OF $\left(X^{\prime}, X_{1}, F\right)$.

CASE 2.1. This Case is verv easy. Let $X^{\prime}:=X, X_{1}:=X_{0}$. We take a divisor $F \subset g^{-1}\left(x_{0}\right)$. Then $X^{\prime}, X_{1}$ and $F$ satisfy the conditions.

CASE 2.2. Let $X_{1}:=X_{0}$. Similarly to the Case 1.3 , we take a normal variety $X^{\prime}$, birational morphism $h: X^{\prime} \rightarrow X$, and the $h$-exceptional divisor $F$. Then $X^{\prime}, X_{1}$ and $F$ satisfy the conditions.

CASE 2.3. Let $X_{1}:=X_{0}$. We choose a curve $C$ in $g^{-1}\left(x_{0}\right)$ and take an embedded resolution of $C, \tilde{h}_{1}: \tilde{X} \rightarrow X$. Let $C^{\prime}$ be the proper transform of $C$. We take the blowing up along $C^{\prime}, \tilde{h}_{2}: X^{\prime} \rightarrow \tilde{X}$. We define $F$ is the exceptional divisor of $\tilde{h}_{2}$. Then $X^{\prime}, X_{1}$ and $F$ satisfy the conditions.

CASE 2.4. Let $\bar{h}: X_{1} \rightarrow X_{0}$ be the minimal resolution of $\left(X_{0}, x_{0}\right)$. Take a divisor $F^{\prime} \subset g^{-1}\left(x_{0}\right)$. By Hironaka [6], we can choose a smooth variety $\tilde{X}$ and a birational morphism $\tilde{h}: \tilde{X} \rightarrow X$ which satisfy the following conditions:

(1) There is a morphism $\bar{g}: \tilde{X} \rightarrow X_{1}$ such that $g \circ \tilde{h}=\bar{h} \circ \bar{g}$.

(2) There is a simple normal crossing divisor $\Sigma$ on $\tilde{X}$ such that

Supp $\Sigma=(\tilde{h}$-exceptional divisor $) \cup\left(\right.$ The proper transform of $\left.F^{\prime}\right)$.

Let $F^{\prime \prime}$ be the proper transform of $F^{\prime}$. If $\bar{g}\left(F^{\prime \prime}\right)$ is one point, then we set $F:=F^{\prime \prime}$ and $X^{\prime}:=\tilde{X}$. If $\bar{g}\left(F^{\prime \prime}\right)$ is a curve $I$, we choose a point $x_{1} \in I$ such that $\bar{g}^{-1}\left(x_{1}\right) \cap F^{\prime \prime}$ is not contained any $\tilde{h}$-exceptional divisor. Then take a curve $C$ such that $\bar{g}(C)=x_{1}$ and $C$ is not contained any $\bar{h}$-exceptional divisors. We construct $X^{\prime}$ and $F$ similarly to Case 2.3 . Since $C$ is not contained any $\bar{h}$-exceptional divisors,

$$
K_{X^{\prime}} \sim_{Q} \bar{h}^{*} K_{X}+F+\text { (other components) }
$$


Other conditions are easily checked.

CASE 2.5. First we construct a normal variety $\tilde{X}$, a birational morphism $\tilde{g}: \tilde{X} \rightarrow X$ and $\tilde{g}$-exceptional divisor $\tilde{F}$ as Case 1.3 . Then we can construct $X^{\prime}$, $X_{1}$ and $F$ similarly to the Case 2.4. We only have to check condition 4. Since $K_{\tilde{X}} \sim_{Q} \tilde{g}^{*} K_{X}+a F+($ other components), $(a \leqq 1)$,

$$
K_{X^{\prime}} \sim_{Q}(\tilde{g} \circ \bar{h}) * K_{X}+(a+1) F+(\text { other components }) .
$$

Other conditions are easily checked.

CASE 2.6. First we construct a normal variety $\tilde{X}$, a birational morphism $\tilde{g}: \tilde{X} \rightarrow X$ and $\tilde{g}$-exceptional divisor $\tilde{F}$ as Case 2.3. Then we can construct $X^{\prime}$, $X_{1}$ and $F$ similarly to the Case 2.4 . We only have to check condition 4 . Since $K_{\tilde{X}} \sim_{Q} \tilde{g}^{*} K_{X}+F+$ (other components),

$$
K_{X^{\prime}} \sim_{\boldsymbol{Q}}(\tilde{g} \circ \bar{h}) * K_{X}+2 F+\text { (other components) } .
$$

Other conditions are easily checked. Then we complete the proof of Proposition of (2).

(3) We take a rational number $\sigma_{1}$ such that $\operatorname{deg} D>\sigma_{1}>1$. Since $x_{0}$ is a smooth point of $X_{0}$, by Lemma 1 we can choose an effective $\boldsymbol{Q}$-divisor $B^{\prime}$ on $X_{0}$ such that $B^{\prime} \sim_{Q} D$ and $\nu_{x_{0}}\left(B^{\prime}\right)>\sigma_{1} /(1-\sigma)$. We define $B:=g^{*} B^{\prime}$. Select a smooth variety $Y$ and a birational morphism $f$ such that the pair $(B, Y, f)$ satisfies the four conditions in (1). Suppose $F$ be an irreducible component of $g^{-1}\left(x_{0}\right)$. Let $E_{1}$ be the proper transform of $F$ by $f$. Then $b_{1}>\sigma_{1} /(1-\sigma), e_{1} \leqq 0$. Thus

$$
c \leqq \frac{e_{1}+1-p_{1}}{b_{1}}<\frac{e_{1}+1}{b_{1}}<\frac{(1-\sigma)}{\sigma_{1}}<\frac{1}{\operatorname{deg} D} .
$$

We complete of the proof of Proposition of (3).

Now we have completed the proof of Theorem 9 .

Q.E.D.

\section{REFERENCES}

[1] E. BRIESKORN, Rationale singularitäten komplexer flächen, Invent. Math., 14 (1968), 336-358.

[2] L. EIN AND R. LAZARSFELD, Global generation of pluricanonical and adjoint linear series on smooth projective threefolds, J. Amer. Math, Soc., 6 (1993), 875-903.

[3] L. Ein, R. Lazarsfeld and V. Masek, Global generation of linear series on terminal threefolds, Internat. J. Math., 6 (1995), 1-18.

[4] T. Fujita, Classification Theories of Polarized Varieties, London Math. Soc. Lecture Notes Ser., 155, Cambridge Univ. Press, 1990. 
[5] R. HARTshorne, Generalized divisors on Gorenstein curves and a theorem of Noether, J. Math. Kyoto Univ., 26 (1986), 375-386.

[6] H. HironaKa, Resolution of singularities of an algebraic variety over a field of characteristic zero, Ann. of Math., 79 (1964), 109-326.

[7] Y. Kawamata, K. Matsuda and K. MatsuKI, Introduction to the minimal model problem, Algebraic Geometry, Sendai, 1985, Adv. Stud. Pure Math., 10, T. Oda ed., Kinokuniya and North-Holland, 1987, 283-360.

[8] Y. Kawamata, Termination of $\log$ flips for algebraic 3-folds, Internat J. Math., 3 (1992), 653-659.

[9] Y. Kawamata, Minimal discrepancy coefficients of terminal singularities in dimension 3, Russian Acad. Sci. Izv. Math., 40 (1993), 193-195, appendix to V. V. Shokurov, 3-folds log flips, Russian Acad. Sci. Izv. Math., 40 (1993), 95-202.

[10] J. Kollár, Effective base point freeness, Math. Ann., 296 (1993), 595-605.

[11] I. REIDER, Vector bundles of rank 2 and linear systems on algebraic surfaces, Ann. of Math., 127 (1988), 309-316.

[12] F. SAKAI, Reider-Serrano's method on normal surfaces, Algebraic Geometory: Proceedings, L'Aquila 1988, Lecture Notes in Math., 1417, Springer, 1990, 301319.

\author{
Graduate School of Mathematical Sciences \\ ThE UNIVERSITY OF TOKYo \\ MEgURO, TOKYo 153 \\ JAPAN \\ e-mail : tyler@ms318sun.ms.u-tokyo.ac.jp
}

